\title{
Artificial Compressibility Method Revisited: Asymptotic Numerical Method for Incompressible Navier-Stokes Equations
}

\author{
Taku Ohwada \\ Department of Aeronautics and Astronautics, Graduate School of Engineering, Kyoto \\ University, Kyoto 606-8501, Japan \\ Pietro Asinari \\ Dipartimento di Energetica, Politecnico di Torino, Torino 10129, Italy
}

\begin{abstract}
The artificial compressibility method for the incompressible Navier-Stokes equations is revived as a high order accurate numerical method (4th order in space and 2nd order in time). Similar to the lattice Boltzmann method, the mesh spacing is linked to the Mach number. The accuracy higher than that of the lattice Boltzmann method is achieved by exploiting the asymptotic behavior of solution of artificial compressibility equations for small Mach numbers and the simple lattice structure of stencil. An easy method for quickening the decay of acoustic waves, which deteriorate the quality of numerical solution, and a simple cure for the checkerboard instability are proposed there. The high performance of the scheme is demonstrated not only for the periodic boundary condition but also for the Dirichlet-type boundary condition.
\end{abstract}

Key words: Incompressible Navier-Stokes Equations, Artificial Compressibility Method, Asymptotic Analysis, Acoustic Wave, Lattice Boltzmann

\section{INTRODUCTION}

Various numerical methods for the incompressible Navier-Stokes equations (INSE) employ Poisson (or Helmholtz) solvers, which require additional computations at each time step. The adoption of an implicit time-integration method is quite natural in this intrinsically implicit approach and the inversion of matrix occupies the major part of computation. The artificial compressibility method (ACM), which was proposed by Chorin [3] in the late '60s of the previous century, is alternative to the intrinsically implicit approach. ACM solves the artificial compressibility equations (ACE), where the solenoidal (divergence-free) condition for the flow velocity field is replaced by a continuity equation with 
the pressure time derivative, which enables us to compute the pressure field evolutionally. Since ACE agree with INSE in the steady case, ACM is said to be principally intended for steady flows and implicit time-integration methods are sometimes introduced. Témam justified ACM mathematically by proving the weak convergence of an ACE solution from an initial data compatible with INSE to the INSE solution for the same initial data in the vanishing limit of Mach number [17]. ACE involve the acoustic (rapidly varying) mode besides the diffusive (slowly varying) mode, the principal part of which is described by INSE. Moise and Ziane employed the renormalization group method in the multi-scale analysis of ACE solution for small Mach numbers and derived the estimate that the error of ACE solution (the deviation from INSE solution) for the flow velocity is bounded by a constant of the order of the Mach number [12].

The lattice Boltzmann method (LBM) is another Poisson-free method for INSE and has been attracting a lot of attention during the last two decades because of its simple numerical algorithm, which is also suitable for parallel computation, and it is now widely employed in various simulations of unsteady complex fluid flows. It deals with the time evolution of the velocity distribution function of "artificial" gas molecules and yields an approximate solution for the time-dependent INSE as the moments of the distribution function. The accuracy of LBM is said to be first order in time and second order in space. For its theoretical justification, we refer the reader to e.g. Refs. [16, 6, 9, 1], where the asymptotic analyses of discrete kinetic equations (or their numerical schemes) for small Knudsen numbers [Chapman-Enskog expansion and Hilbert expansion (diffusive scaling)] are carried out. These asymptotic analyses indirectly show the relation between LBM and ACM. Chapman-Enskog expansion derives INSE via ACE as the leading equation system in the $\mathrm{Ma}^{2}$ expansion for $\mathrm{ACE}$ ( $\mathrm{Ma}$ is the Mach number) and ACE is also recovered by summing up the equation systems derived by Hilbert expansion. Since LBM computes the distribution function of gas molecules, it has been expected to have potential abilities to deal with a rarefied regime beyond Navier-Stokes. In our previous paper [1], we examined this possibility and arrived at the conclusion that the usual compact stencils, such as D2Q9 and D3Q15, are not sufficient for the realization of physically correct high order stress and heat flow. On the other hand, the kinetic formulation has a clear meaning in the compressible case. The linearity of convective term of kinetic equation drastically simplifies the theory of approximate Riemann solver, which is emloyed in most of shock-capturing schemes (see e.g. Ref. [13] and the references therein). This naturally arouses a question concerning the advantage of kinetic formulation in the incompressible case. Of course, one of its advantages must be found in the performance at least. Although comparisons of ACM and LBM have been made in the literature (e.g. Ref. [7]), however, it does not seem to be necessary to draw hasty conclusions at the present stage, since LBM is still developing and it is not too much to say that the potential ability of ACM has not yet been explored fully.

The present study concerns the exploration of potential ability of ACM. Since LBM deals with the passage of $\mathrm{Kn} \sim \mathrm{Ma} \sim \epsilon \rightarrow 0$, where $\mathrm{Kn}$ and $\epsilon$ are the Knudsen number and the mesh spacing, respectively, we regard ACM as an 
asymptotic numerical method dealing with the passage of $\mathrm{Ma} \sim \epsilon \rightarrow 0$ and look for its potential ability in this direction. Since the simple lattice structure of stencil is considered to be one of the reasons for the success of LBM, we will also make use of it. The remainder of the present paper is organized as follows. The asymptotic behavior of ACE solution for small Mach numbers will be studied in Sec. 2, where the construction of diffusive mode, the excitation of acoustic mode due to incomplete initial data, a new dissipation mechanism to kill the acoustic mode quickly, and a strategy of high order accurate computation of INSE will be explained. We aim to solve INSE within the error of $O\left(\mathrm{Ma}^{4}\right)$ by computing two ACE solutions for different Mach numbers under the same resolution, which is different from the conventional Richardson extrapolation. The elimination of acoustic mode will be one of the key issues for the realization of high accuracy. In Sec. 3, the basic design of the numerical scheme will be prepared. A prototype scheme for a linear 2D model PDE system will be studied and a simple cure for the checkerboard instability will be proposed. The prototype scheme will be extended to the case of ACE in Sec. 4, where high order accurate treatment of boundary condition will be explained in detail. The developed method will be tested in Sec. 5. A comparison will also be made with LBM there.

\section{THEORY}

\subsection{IBVP for INSE}

INSE in the dimensionless form are expressed as

$$
\begin{gathered}
\frac{\partial u_{i}}{\partial x_{i}}=0 \\
\frac{\partial u_{i}}{\partial t}+u_{j} \frac{\partial u_{i}}{\partial x_{j}}+\frac{\partial P}{\partial x_{i}}-\nu \frac{\partial^{2} u_{i}}{\partial x_{j}^{2}}=f_{i}
\end{gathered}
$$

where $x_{i}, t, u_{i}, P, f_{i}$, and $\nu$ are the space coordinates, time, flow velocity, pressure, external force, and inverse Reynolds number, respectively. We consider the problem in a bounded domain $\Omega$ with the Dirichlet-type boundary condition:

$$
u_{i}\left(t, x_{j}\right)=g_{i}\left(t, x_{j}\right) \quad x_{j} \in \partial \Omega .
$$

The vector $g_{i}$ must satisfy the compatibility condition

$$
\int_{\partial \Omega} g_{i} n_{i} d S=0,
$$

where $n_{i}$ is the unit vector normal to the boundary pointing in the outward direction. We consider the case where the initial velocity field $u_{i}\left(t=0, x_{j}\right)=$ $u_{i}^{*}\left(x_{j}\right)$ is compatible with the governing equation system and the boundary condition, i.e.

$$
\frac{\partial u_{i}^{*}}{\partial x_{i}}=0
$$




$$
u_{i}^{*}\left(x_{j}\right)=g_{i}\left(t=0, x_{j}\right) \quad x_{j} \in \partial \Omega .
$$

The pressure field $P\left(t, x_{j}\right)$ is determined from the velocity field $u_{i}\left(t, x_{j}\right)$ as a solution of the Neumann problem for the Poisson equation derived from the momentum equation (2). The boundary condition for $P$ is obtained by multiplying both hand sides of Eq. (2) by $n_{i}$. The initial pressure field $P\left(t=0, x_{i}\right)=P^{*}\left(x_{i}\right)$ is generated from $u_{i}^{*}$ by the same recipe. We impose

$$
\int_{\Omega} P\left(t, x_{k}\right) d \boldsymbol{V}=0
$$

in order to make $P$ unique.

\section{2. $A C E$}

The original ACM employs the following artificial continuity equation

$$
k \frac{\partial P}{\partial t}+\frac{\partial u_{i}}{\partial x_{i}}=0
$$

instead of the solenoidal condition, where $k$ is a positive constant. In the present study, we consider the case of $0<k \ll 1$. Eqs. (2) and (8) constitute ACE. We will consider the problem of ACE under the boundary condition (3). By imposing

$$
\int_{\Omega} P\left(0, x_{i}\right) d \boldsymbol{V}=0
$$

the condition (7) is always satisfied owing to the compatibility condition (4).

\subsection{Diffusive mode and acoustic mode}

As mentioned previously, ACE involve the diffusive mode and the acoustic mode. We will sketch these two modes as the preparation for the later discussion.

We assume that both modes are slowly varying with respect to space:

$$
\frac{\partial P}{\partial x_{j}} \sim O(P), \quad \frac{\partial u_{i}}{\partial x_{j}} \sim O\left(u_{i}\right)
$$

The diffusive mode is slowly varying with respect to time as well:

$$
\frac{\partial P}{\partial t} \sim O(P), \quad \frac{\partial u_{i}}{\partial t} \sim O\left(u_{i}\right)
$$

Assuming $P \sim O(1)$ and $u_{i} \sim O(1)$, i.e.

$$
\begin{gathered}
P \sim \frac{\partial P}{\partial x_{i}} \sim \frac{\partial P}{\partial t} \sim O(1), \\
u_{i} \sim \frac{\partial u_{i}}{\partial x_{j}} \sim \frac{\partial u_{i}}{\partial t} \sim O(1),
\end{gathered}
$$

and neglecting the pressure time derivative multiplied by the small parameter $k$, we have the solenoidal condition from the artificial continuity equation (8). 
The momentum equation (2) remains as it is. The resulting equation system is INSE.

The acoustic mode solution is rapidly varying with respect to time. Neglecting the nonlinear term, the force term, and the viscous term in the momentum equation, we have the simplified $\mathrm{ACE}$ :

$$
\begin{aligned}
& k \frac{\partial P}{\partial t}+\frac{\partial u_{i}}{\partial x_{i}}=0, \\
& \frac{\partial u_{i}}{\partial t}+\frac{\partial P}{\partial x_{i}}=0 .
\end{aligned}
$$

The wave equation for $P$ with the propagation speed $k^{-1 / 2}$ is derived from the above equations, which shows that the characteristic time of the acoustic mode is $O\left(k^{1 / 2}\right)$. We introduce the new time variable $\tau$ defined by

$$
\tau=k^{-1 / 2} t
$$

and rewrite the above equation system using $\tau$ :

$$
\begin{gathered}
k^{1 / 2} \frac{\partial P}{\partial \tau}+\frac{\partial u_{i}}{\partial x_{i}}=0, \\
k^{-1 / 2} \frac{\partial u_{i}}{\partial \tau}+\frac{\partial P}{\partial x_{i}}=0 .
\end{gathered}
$$

Since

$$
\frac{\partial P}{\partial \tau} \sim O(P), \quad \frac{\partial u_{i}}{\partial \tau} \sim O\left(u_{i}\right)
$$

we notice $P \sim k^{-1 / 2} u_{i}$ from the balance of terms in the above equations. The magnitude of acoustic mode for the pressure is much larger than that for the flow velocity.

\subsection{Construction of diffusive mode}

We will construct the slowly varying solution of ACE (2) and (8) satisfying the boundary condition (3) under the assumptions (10) and (11). We express the slowly varying solution as $\left(u_{i S}, P_{S}\right)$ and expand it into the power series of $k$ :

$$
\begin{gathered}
u_{i S}=u_{i S 0}+k u_{i S 1}+k^{2} u_{i S 2}+\cdots, \\
P_{S}=P_{S 0}+k P_{S 1}+k^{2} P_{S 2}+\cdots .
\end{gathered}
$$

It should be noted that the above expansion is nothing more than the wellknown $\mathrm{Ma}^{2}$ expansion (the sound speed corresponds to $k^{-1 / 2}$ ). The condition (7) is expanded accordingly:

$$
\int_{\Omega} P_{S m}\left(t, x_{k}\right) d \boldsymbol{V}=0 \quad(m=0,1,2,3, \cdots) .
$$


We assume

$$
\begin{gathered}
u_{i S m} \sim \frac{\partial u_{i S m}}{\partial x_{j}} \sim \frac{\partial u_{i S m}}{\partial t} \sim O(1) \quad(m=0,1,2, \cdots), \\
\frac{\partial P_{S m}}{\partial x_{i}} \sim \frac{\partial P_{S m}}{\partial t} \sim O(1) \quad(m=0,1,2, \cdots),
\end{gathered}
$$

which correspond to the assumptions (10) and (11). Substituting the above expansion into Eqs. (2) and (8) and equating the terms of the same order of power of $k$, we have the following sequence of the equation systems:

$$
\begin{gathered}
\frac{\partial u_{i S 0}}{\partial x_{i}}=0 \\
\frac{\partial u_{i S 0}}{\partial t}+u_{j S 0} \frac{\partial u_{i S 0}}{\partial x_{j}}+\frac{\partial P_{S 0}}{\partial x_{i}}-\nu \frac{\partial^{2} u_{i S 0}}{\partial x_{j}^{2}}=f_{i} \\
\frac{\partial u_{i S 1}}{\partial x_{i}}+\frac{\partial P_{S 0}}{\partial t}=0 \\
\frac{\partial u_{i S 1}}{\partial t}+\mathcal{L}_{i}\left[u_{k S 1}, P_{S 1} ; u_{k S 0}\right]=0 \\
\frac{\partial u_{i S 2}}{\partial x_{i}}+\frac{\partial P_{S 1}}{\partial t}=0 \\
\frac{\partial u_{i S 2}}{\partial t}+\mathcal{L}_{i}\left[u_{k S 2}, P_{S 2} ; u_{k S 0}\right]+u_{j S 1} \frac{\partial u_{i S 1}}{\partial x_{j}}=0 \\
\ldots
\end{gathered}
$$

where

$$
\mathcal{L}_{i}\left[u_{k}, P ; V_{k}\right] \equiv V_{j} \frac{\partial u_{i}}{\partial x_{j}}+u_{j} \frac{\partial V_{i}}{\partial x_{j}}+\frac{\partial P}{\partial x_{i}}-\nu \frac{\partial^{2} u_{i}}{\partial x_{j}^{2}} .
$$

The leading equation system is INSE and inhomogeneous Oseen-type equation systems follow. The boundary condition is expanded accordingly:

$$
\begin{gathered}
u_{i S 0}\left(t, x_{j}\right)=g_{i}\left(t, x_{j}\right) \quad x_{j} \in \partial \Omega, \\
u_{i S m}\left(t, x_{j}\right)=0 \quad x_{j} \in \partial \Omega \quad(m \geq 1) .
\end{gathered}
$$

We can, in principle, construct the diffusive mode solution by solving the above equation system from the lowest order with appropriate initial data. Let $\left(u_{i S m}^{*}, P_{S m}^{*}\right)(m=0,1,2,3, \cdots)$ be the initial data for $\left(u_{i S m}, P_{S m}\right)$. As the initial data $\left(u_{i S 0}^{*}, P_{S 0}^{*}\right)$, we can choose the same one as that for INSE:

$$
\begin{aligned}
& u_{i S 0}^{*}=u_{i}^{*} . \\
& P_{S 0}^{*}=P^{*} .
\end{aligned}
$$

Since the divergence of $u_{i S 1}$ is determined by the time derivative of $P_{S 0}$ [Eq. (21)], we cannot give the initial data $\left(u_{i S 1}^{*}, P_{S 1}^{*}\right)$ arbitrarily. The recipe of construction 
of $\left(u_{i S 1}^{*}, P_{S 1}^{*}\right)$ is as follows. We first construct $u_{i S 1}^{*}$ as the sum of an irrotational vector field $d_{i}$ and a solenoidal vector field $s_{i}$ :

$$
u_{i S 1}^{*}=d_{i}+s_{i}
$$

The vector field $d_{i}$ is defined by

$$
d_{i}=\frac{\partial \phi}{\partial x_{i}}
$$

where the potential $\phi$ is a solution of the Poisson equation

$$
\frac{\partial^{2} \phi}{\partial x_{i}^{2}}=-\left.\frac{\partial P_{S 0}}{\partial t}\right|_{t=0}
$$

under the Neumann-type boundary condition

$$
n_{i} \frac{\partial \phi}{\partial x_{i}}=0 \quad x_{i} \in \partial \Omega
$$

The solenoidal vector field $s_{i}$ must satisfy $s_{i}=-d_{i}$ at the boundary $\partial \Omega$. We refer the reader to e.g. Ref. [10] for the details of construction of $s_{i} ; s_{i}$ is not determined uniquely since there are infinite solenoidal vector fields satisfying the homogeneous boundary condition. Once $u_{i S 1}^{*}$ is determined, $P_{S 1}^{*}$ is computed in the following way. Taking the divergence of the momentum equation (22), we have the Poisson equation for $P_{S 1}^{*}$ :

$\frac{\partial^{2} P_{S 1}^{*}}{\partial x_{i}^{2}}=-\left(\left.\frac{\partial \Theta}{\partial t}\right|_{t=0}+\frac{\partial u_{j S 0}^{*}}{\partial x_{i}} \frac{\partial u_{i S 1}^{*}}{\partial x_{j}}+\left.u_{j S 0}^{*} \frac{\partial \Theta}{\partial x_{j}}\right|_{t=0}+\frac{\partial u_{j S 1}^{*}}{\partial x_{i}} \frac{\partial u_{i S 0}^{*}}{\partial x_{j}}\right)+\left.\nu \frac{\partial^{2} \Theta}{\partial x_{k}^{2}}\right|_{t=0}$,

where

$$
\Theta=-\frac{\partial P_{S 0}}{\partial t}
$$

The Neumann boundary condition for Eq. (34) is also supplied from the momentum equation (22):

$$
n_{i} \frac{\partial P_{S 1}^{*}}{\partial x_{i}}=n_{i}\left[\nu \frac{\partial^{2} u_{i S 1}^{*}}{\partial x_{k}^{2}}-u_{j S 0}^{*} \frac{\partial u_{i S 1}^{*}}{\partial x_{j}}\right] \quad x_{i} \in \partial \Omega,
$$

where the boundary condition for $u_{i S 1}$, Eq. (27), is taken into account. Then, $P_{S 1}^{*}$ is determined as the solution of the above BVP under the condition (16). We notice that the construction of $u_{i S 1}^{*}$ and $P_{S 1}^{*}$ requires the information of the time derivatives $\partial_{t} P_{S 0}$ and $\partial_{t t} P_{S 0}$ at $t=0$. The construction of $\left(u_{i S m}, P_{S m}\right)$ $(m=2,3,4, \cdots)$ is similar and it requires higher order time derivatives for the INSE solution. 


\subsection{Excitation of acoustic mode}

In the case of complete initial data for the diffusive mode

$$
u_{i S}^{*}=u_{i S 0}^{*}+k u_{i S 1}^{*}+k^{2} u_{i S 2}^{*}+\cdots, \quad P_{S}^{*}=P_{S 0}^{*}+k P_{S 1}^{*}+k^{2} P_{S 2}^{*}+\cdots,
$$

ACE yields only the diffusive mode in principle. In the case of truncated initial data, however, the solution of ACE is not launched smoothly along the trajectory of diffusive mode and the gap excites the acoustic mode. We will estimate the magnitude of the excited acoustic mode in the simplest case where the initial data is the one for INSE:

$$
\begin{aligned}
& u_{i}(t=0)=u_{i S 0}^{*} \equiv u_{i}^{*}, \\
& P(t=0)=P_{S 0}^{*} \equiv P^{*} .
\end{aligned}
$$

Rewriting Eqs. (2) and (8) as those for the perturbation from the INSE solution, $w_{i} \equiv u_{i}-u_{i S 0}$ and $q \equiv P-P_{S 0}$, we have

$$
\begin{gathered}
\frac{\partial w_{i}}{\partial t}+\mathcal{L}_{i}\left[w_{k}, q ; u_{k S 0}\right]+w_{j} \frac{\partial w_{i}}{\partial x_{j}}=0, \\
k \frac{\partial q}{\partial t}+\frac{\partial w_{j}}{\partial x_{j}}=-k \frac{\partial P_{S 0}}{\partial t} .
\end{gathered}
$$

The boundary condition and the initial condition for $\left(w_{i}, q\right)$ are

$$
w_{i}=0 \quad x_{i} \in \partial \Omega ; \quad\left(w_{i}, q\right)=(\mathbf{0}, 0) \quad(t=0) .
$$

From Eqs. (39) and (40), we have

$$
\frac{\partial q}{\partial t}=-\frac{\partial P_{S 0}}{\partial t} \quad(t=0),
$$

which shows that $\partial_{t} q$ is $O(1)$ at $t=0$. The right hand side of Eq. (39) acts as an initial impact on $q$ and it excites the acoustic mode. Let us estimate the magnitude of the acoustic mode. From the discussion in Sec. 2.3, the characteristic time of acoustic mode is $O\left(k^{1 / 2}\right)$ under the assumption that the wave-length is $O(1)$. We introduce the time variable $\tau$, which is defined by Eq. (12). From $\partial_{\tau} q \sim O(q)$ and $\partial_{t} q \sim k^{-1 / 2} \partial_{\tau} q \sim O(1)$, we have $q \sim O\left(k^{1 / 2}\right)$. If we require the balance of the term $\partial w_{j} / \partial x_{j}$ with the other terms in Eq. (39), we have $w_{i} \sim O(k)$. This is a more optimistic estimate than that obtained by the renormalization group method in Ref. [12], i.e. $\left|w_{i}\right| \lesssim O\left(k^{1 / 2}\right)$.

\subsection{Suppression of acoustic mode}

The acoustic mode excited by the initial impact deteriorates the quality of ACE solution as an approximate solution of INSE especially for the pressure. The employment of high order initial data for the diffusive mode reduces the initial impact and the magnitude of the acoustic wave decreases accordingly. A simple discussion similar to the previous subsection leads to the conclusion that 
the magnitude of acoustic mode is reduced from $O(k)$ to $O\left(k^{2}\right)$ for the velocity and from $O\left(k^{1 / 2}\right)$ to $O\left(k^{3 / 2}\right)$ for the pressure by changing the initial data from $\left(u_{i S 0}^{*}, P_{S 0}^{*}\right)$ to $\left(u_{i S 0}^{*}+k u_{i S 1}^{*}, P_{S 0}^{*}+k P_{S 1}^{*}\right)$. We refer the reader to Ref. [14] for the numerical example of suppression of acoustic mode by this approach. However, as seen previously, this requires the information of $\partial_{t} P_{S 0}$ and $\partial_{t t} P_{S 0}$ at $t=0$ and even the construction of the initial data $\left(u_{i S 1}^{*}, P_{S 1}^{*}\right)$ is cumbersome in general. The second possibility is the introduction of dissipation term into the continuity equation:

$$
k\left(\frac{\partial P}{\partial t}+\gamma P\right)+\frac{\partial u_{i}}{\partial x_{i}}=0
$$

where $\gamma$ is a positive function of $t$ and is of the order of unity. In the following discussions, we will treat it as a constant for simplicity.

We rewrite Eqs. (2) and (41) using $W_{i}=\left(u_{i}-u_{i S 0}\right) k^{-1}$ and $Q=(P-$ $\left.P_{S 0}\right) k^{-1 / 2}$ :

$$
\begin{gathered}
\frac{\partial W_{i}}{\partial \tau}+\frac{\partial Q}{\partial x_{i}}+k^{1 / 2}\left(u_{j S 0} \frac{\partial W_{i}}{\partial x_{j}}+W_{j} \frac{\partial u_{i S 0}}{\partial x_{j}}+k W_{j} \frac{\partial W_{i}}{\partial x_{j}}\right)=k^{1 / 2} \nu \frac{\partial^{2} W_{i}}{\partial x_{k}^{2}} \\
\frac{\partial Q}{\partial \tau}+\gamma k^{1 / 2} Q+\frac{\partial W_{i}}{\partial x_{i}}+\left(\frac{\partial P_{S 0}}{\partial t}+\gamma P_{S 0}\right)=0
\end{gathered}
$$

In order to illustrate the role of the new dissipation terms, we consider the simplest case of $u_{i S 0}=0$ and neglect the nonlinear term $k^{3 / 2} W_{j} \partial W_{i} / \partial x_{j}$ as in the case of the derivation of the equation of sound waves. Then, we obtain the dissipative wave equation for $Q$ from the above equation system:

$$
\begin{aligned}
\frac{\partial^{2} Q}{\partial \tau^{2}} & -(1+\gamma \nu k) \frac{\partial^{2} Q}{\partial x_{k}^{2}}+\gamma k^{1 / 2} \frac{\partial Q}{\partial \tau}-k^{1 / 2} \nu \frac{\partial^{3} Q}{\partial x_{k}^{2} \partial \tau} \\
& =-k^{1 / 2}\left(\frac{\partial^{2} P_{S 0}}{\partial t^{2}}+\gamma \frac{\partial P_{S 0}}{\partial t}\right)+\nu k^{1 / 2} \frac{\partial^{2}}{\partial x_{k}^{2}}\left(\frac{\partial P_{S 0}}{\partial t}+\gamma P_{S 0}\right) .
\end{aligned}
$$

The right hand side acts as slowly varying external force. The fourth term on the left hand side is the viscous damping term. The third term on the same side, which is multiplied by $\gamma$, acts like a dashpot in a simple mechanical oscillation system. While the viscous damping term does not work well for small $\nu$ (large Reynolds number), this newly introduced dissipation term works irrespective of the magnitude of $\nu$. While the work of viscous damping term increases as the wave number increases, the new damping term works uniformly for the wave number. Incidentally, the bulk viscosity terms is sometimes introduced in the momentum equation for the suppression of the acoustic mode (see e.g. Ref. [15]) and this idea is also inherited in $\operatorname{LBM}[5,11]$. Indeed, the introduction of bulk viscosity increases the coefficient of the viscous damping term in Eq. (42). In the present study, the damping for high wave numbers is treated in connection with the suppression of checkerboard instability (Sec. 3.2).

The introduction of the new damping term modifies the governing equation systems for the diffusive mode from the order of $k$; Eqs. (21) and (23) become

$$
\frac{\partial u_{i S 1}}{\partial x_{i}}+\frac{\partial P_{S 0}}{\partial t}+\gamma P_{S 0}=0
$$




$$
\frac{\partial u_{i S 2}}{\partial x_{i}}+\frac{\partial P_{S 1}}{\partial t}+\gamma P_{S 1}=0,
$$

respectively. The initial data for $\left(u_{i S m}, P_{S m}\right)$ can be constructed in the same way. Incidentally, the condition (7) still follows from Eq. (9).

\subsection{Main assumption and strategy}

Let us consider the solution of IBVP for Eqs. (2) and (41) with the initial data (36) and (37) and the boundary condition (3). Because of the incomplete initial data, the acoustic mode is inevitably excited. The acoustic mode is weakened by the viscous damping term as time advances and the decay is further accelerated by the newly implemented dashpot-type damping term. After the extinction of the acoustic mode, the behavior of solution is described by using the single time scale for slowly varying. We recall that there is only one parameter in this IBVP, i.e. $k$. We sssume the form of Eqs. (14) and (15) as the anzats of asymptotic solution for small $k$ after the extinction of the acoustic mode. This is the key assumption in the present study. The leading term $\left(u_{i S 0}, P_{S 0}\right)$ in the $k$ expansion is the desired INSE solution for the initial data (36) and (37), which follows from the convergence theorem for $k \rightarrow 0$ [17]. The next order term $\left(u_{i S 1}, P_{S 1}\right)$ is governed by the inhomogeneous Oseen-type equation system, i.e. Eqs. (21) and (22). Then, we can excavate the desired INSE solution within the error of $O\left(k^{2}\right)$ from two solutions of ACE for different values of $k$. That is, the leading error $\left(k u_{i S 1}, k P_{S 1}\right)$ can be eliminated by computing a suitable linear combination of these two solutions.

The above scenario is not clear from the dynamical point of view, however. Let us trace the diffusive mode solution back to $t=0$. We recall that there is a freedom in the choice of solenoidal part of the initial velocity field $u_{i S 1}^{*}$ (Sec. 2.4). While the anzats implies that $u_{i S 1}$ (and therefore $u_{i S 1}^{*}$ ) is unique irrespective of the value of $k$, its theoretical assurance is not presently available to the best of authors' knowledge. The scenario would be supported numerically.

ACM is intrinsically explicit and the adoption of an explicit time-integration method is quite natural. Then, the time step $\Delta t$ will be subject to the acoustic CFL condition $\Delta t \lesssim k^{1 / 2} \epsilon$, where $\epsilon$ is the mesh spacing and $k^{-1 / 2}$ is the sound speed of ACE. Another time step restriction is the diffusive CFL condition $\Delta t \lesssim \epsilon^{2} / \nu$. The former condition is related to the intrinsic error of ACE. Small $k$ is preferable for the accuracy and vice versa for the efficiency of numerical computation. The latter condition does not become more serious than the former one while $\nu$ is not large, i.e. $\nu \lesssim 1$. We link the mesh spacing $\epsilon$ and the compressibility parameter $k$ by the relation

$$
k=\beta \epsilon^{2},
$$

where $\beta$ is a positive constant of the order of unity. Then, the acoustic CFL condition is rewritten as $\Delta t \lesssim \epsilon^{2}$, which corresponds to the diffusive CFL condition for $\nu \sim 1$.

Our strategy is rephrased as follows. We will obtain the solution of INSE within the error of $O\left(\epsilon^{4}\right)$ from two numerical solutions of ACE for different 
values of $\beta$ under the same resolution $\epsilon$ by making use of the linearity of the leading error in $\beta$. The behavior of numerical solution depends not only on the parameter of equation system but also on the discretization error. For the realization of the above scenario, the discretization error must not alter the form of the first two equation systems of the diffusive mode. In the next subsection, we will study the influence of the discretization error on the diffusive mode solution.

\subsection{Formal asymptotic analysis of numerical scheme for small $\epsilon$}

In the error analysis of a finite difference scheme, we tacitly assume that the discrete numerical data $\left[u_{i}\right.$ and $P$ at $\left(t, x_{1}, x_{2}, x_{3}\right)=\left(n \Delta t, r_{1} \epsilon, r_{2} \epsilon, r_{3} \epsilon\right)(n=$ $\left.0,1,2,3, \cdots, r_{i}=0,1,2,3, \cdots\right)$ in our problem] behave like the samples taken from a smooth mother function (functions), which depends on the discretization parameter, such as the mesh spacing $\epsilon$. The asymptotic behavior of the mother function for small $\epsilon$ provides the information of consistency and accuracy for the numerical method. Suppose that the mother function is expanded into the power series of $\epsilon$. The coefficient functions at the leading order must satisfy the target equation (system). If the coefficient functions at the next order are governed by an equation (system) which does not allow the null solution, the numerical method is judged to be at most first order accurate in $\epsilon$. If it does, the numerical method has the possibility to yield a second order accurate solution. In other words, the asymptotic analysis of numerical scheme provides the governing equation (system) for the discretization error. Let us go back to our problem. The error of ACM consists of the discretization error and the intrinsic error of ACE, i.e. the error due to the compressibility, as in the case of LBM. We refer the reader to Ref. [8] for the details of the asymptotic theory for finite difference methods and Ref. [9] for its application to LBM.

We investigate the asymptotic behavior of numerical solution for ACE (2) and (41) with $k=\beta \epsilon^{2}$ for small mesh spacing $\epsilon$ under the assumption that the numerical solution is slowly varying. We will employ only the formal accuracy of the scheme in the following analysis; the explicit form of the scheme is not necessary. We first deal with the space variables continuously and investigate the truncation error with respect to time. We consider the case where the formal accuracy of the time integration method is 2nd order. We express the time step $\Delta t$ as $b \epsilon^{2}$, where $b$ is a positive constant of $O(1)$, and write the mother functions for the fixed values of $\beta$ and $b$ as $\hat{u}_{i}\left(t, x_{i} ; \epsilon^{2}\right)$ and $\hat{P}\left(t, x_{i} ; \epsilon^{2}\right)$. The numerical solution at $t=n b \epsilon^{2}(n=0,1,2,3, \cdots)$ is expressed as $\hat{u}_{i}\left(n b \epsilon^{2}, x_{i} ; \epsilon^{2}\right)$ and $\hat{P}\left(n b \epsilon^{2}, x_{i} ; \epsilon^{2}\right)$. Since the time-integration is second order accurate, the mother functions for the finite difference scheme must satisfy

$$
\begin{aligned}
\frac{\partial \hat{P}}{\partial t} & +\frac{b \epsilon^{2}}{2} \frac{\partial^{2} \hat{P}}{\partial t^{2}}+\cdots=-\left[\gamma \hat{P}+\frac{1}{k} \frac{\partial \hat{u}_{j}}{\partial x_{j}}\right] \\
& +\frac{b \epsilon^{2}}{2}\left[\gamma^{2} \hat{P}+\frac{1}{k} \frac{\partial}{\partial x_{j}}\left(\gamma \hat{u}_{j}+m_{j}-f_{j}\right)\right]+O\left(\epsilon^{4}\right),
\end{aligned}
$$




$$
\begin{aligned}
\frac{\partial \hat{u}_{i}}{\partial t} & +\frac{b \epsilon^{2}}{2} \frac{\partial^{2} \hat{u}_{i}}{\partial t^{2}}+\cdots=-m_{i}+f_{i} \\
& +\frac{b \epsilon^{2}}{2}\left[\left(m_{j}-f_{j}\right) \frac{\partial \hat{u}_{i}}{\partial x_{j}}+\hat{u}_{j} \frac{\partial}{\partial x_{j}}\left(m_{i}-f_{i}\right)+\gamma \frac{\partial \hat{P}}{\partial x_{i}}\right. \\
& \left.+\frac{1}{k} \frac{\partial^{2} \hat{u}_{j}}{\partial x_{i} \partial x_{j}}-\nu \frac{\partial^{2}}{\partial x_{j}^{2}}\left(m_{i}-f_{i}\right)+\frac{\partial f_{i}}{\partial t}\right]+O\left(\epsilon^{4}\right),
\end{aligned}
$$

at each discrete point $t=n b \epsilon^{2}(n=0,1,2,3, \cdots)$, where

$$
m_{i}=\hat{u}_{j} \frac{\partial \hat{u}_{i}}{\partial x_{j}}+\frac{\partial \hat{P}}{\partial x_{i}}-\nu \frac{\partial^{2} \hat{u}_{i}}{\partial x_{j}^{2}}
$$

We expand $\hat{P}$ and $\hat{u}_{i}$ into the power series of $\epsilon^{2}$ :

$$
\begin{aligned}
& \hat{P}=\hat{P}^{(0)}+\hat{P}^{(1)} \epsilon^{2}+\hat{P}^{(2)} \epsilon^{4}+\cdots, \\
& \hat{u}_{i}=u_{i}^{(0)}+\hat{u}_{i}^{(1)} \epsilon^{2}+\hat{u}_{i}^{(2)} \epsilon^{4}+\cdots,
\end{aligned}
$$

where $\hat{P}^{(m)}$ and $\hat{u}_{i}^{(m)}(m=0,1,2,3, \cdots)$ are the function of $t$ and $x_{i}$. Substituting the expansion (49) and (50) into Eqs. (46) and (47), noting $k=\beta \epsilon^{2}$, and assuming the slowly varying mode, i.e. $\partial_{\alpha} \hat{P}^{(m)} \sim \partial_{\alpha} \hat{u}_{i}^{(m)} \sim O(1)\left(\alpha=t, x_{i}\right)$, we have the sequence of the equation systems for $\left(\hat{u}_{i}^{(m)}, \hat{P}^{(m)}\right)(m=0,1,2, \cdots)$. The leading equation system is, of course, INSE:

$$
\begin{gathered}
\frac{\partial \hat{u}_{i}^{(0)}}{\partial x_{i}}=0 \\
\frac{\partial \hat{u}_{i}^{(0)}}{\partial t}+\hat{u}_{j}^{(0)} \frac{\partial \hat{u}_{i}^{(0)}}{\partial x_{j}}+\frac{\partial \hat{P}^{(0)}}{\partial x_{i}}-\nu \frac{\partial^{2} \hat{u}_{i}^{(0)}}{\partial x_{j}^{2}}-f_{i}=0,
\end{gathered}
$$

and the inhomogeneous Oseen-type equation systems follow:

$$
\begin{gathered}
\frac{\partial u_{i}^{(1)}}{\partial x_{i}}+\beta\left(\frac{\partial \hat{P}^{(0)}}{\partial t}+\gamma \hat{P}^{(0)}\right)=0, \\
\frac{\partial \hat{u}_{i}^{(1)}}{\partial t}+\mathcal{L}_{i}\left[\hat{u}_{k}^{(1)}, \hat{P}^{(1)} ; \hat{u}_{k}^{(0)}\right]=0, \\
\frac{\partial \hat{u}_{i}^{(2)}}{\partial x_{i}}+\beta\left(\frac{\partial P^{(1)}}{\partial t}+\gamma \hat{P}^{(1)}\right)=0, \\
\frac{\partial \hat{u}_{i}^{(2)}}{\partial t}+\mathcal{L}_{i}\left[\hat{u}_{k}^{(2)}, \hat{P}^{(2)} ; \hat{u}_{k}^{(0)}\right]+\hat{u}_{j}^{(1)} \frac{\partial \hat{u}_{i}^{(1)}}{\partial x_{j}}+I_{N}=0,
\end{gathered}
$$

where the discretization error appears as the term $I_{N}$, the explicit form of which depends on the time-integration method. In the case of first order accurate timeintegration method, the discretization errors appear from the equation system 
for $\left(\hat{u}_{i}^{(1)}, \hat{P}^{(1)}\right)$ as the source terms, which are not proportional to $\beta$. Therefore, the time-integration method must be at least second order accurate.

Up to now, we have not yet taken into account of the spatial discretization error. It can be treated in the above analysis as the additional source term. If the discretization error of $\operatorname{div} \boldsymbol{u}$ is $O\left(\epsilon^{2}\right)$, it appears in Eq. (53). If the error of $m_{i}$ is $O\left(\epsilon^{2}\right)$, it appears in Eq. (54). These errors are not proportional to $\beta$ and break down the linearity of $\left(\hat{u}_{i}^{(1)}, \hat{P}^{(1)}\right)$ in $\beta$. Our strategy survives if the spatial discretization is fourth order accurate; the equation systems for $\left(\hat{u}_{i}^{(m)}, \hat{P}^{(m)}\right)$ $(m=2,3,4, \cdots)$ are altered in this case.

Precisely speaking, we cannot regard the above relations (51)-(56) as PDE systems yet; they hold only at the discrete points $(x, y, t)=\left(i \epsilon, j \epsilon, n b \epsilon^{2}\right)(i=$ $0,1,2,3, \cdots, j=0,1,2,3, \cdots, n=0,1,2,3, \cdots)$. Since the set of discrete points becomes dense in $\Omega \times[0 \leq t]$, they can be regarded as PDEs. We refer the reader to Ref. [8] for its technical discussion.

\section{BASIC DESIGN OF NUMERICAL SCHEME}

\subsection{Prototype scheme}

We prepare the basic design for the numerical scheme of ACE using the following 2D Stokes-type ACE:

$$
\begin{gathered}
\frac{\partial P}{\partial t}+c^{2}\left(\frac{\partial u}{\partial x}+\frac{\partial v}{\partial y}\right)=0 \\
\frac{\partial u}{\partial t}+\frac{\partial P}{\partial x}=\nu\left(\frac{\partial^{2} u}{\partial x^{2}}+\frac{\partial^{2} u}{\partial y^{2}}\right), \\
\frac{\partial v}{\partial t}+\frac{\partial P}{\partial y}=\nu\left(\frac{\partial^{2} u}{\partial x^{2}}+\frac{\partial^{2} u}{\partial y^{2}}\right),
\end{gathered}
$$

where $x_{1}, x_{2}, u_{1}$, and $u_{2}$ are rewritten as $x, y, u$, and $v$, respectively, and $c$ is a positive constant corresponding to the sound speed. We have seen in Sec. 2.7 that the second order accuracy in time is one of the necessary conditions for the realization of our scenario. We adopt a two step midpoint rule (RK-2) as the time-integration method. The notation concerning the spatial discretization is summarized as follows. We introduce the grid system of the uniform spacing $\epsilon,\left(x^{(i)}, y^{(j)}\right)=(i \epsilon, j \epsilon)[i=0,1,2, \cdots, j=0,1,2, \cdots]$. We employ the finite difference operators $\delta_{x}, \delta_{y}, D_{x}$, and $D_{y}, \delta_{x x}, \delta_{y y}, \delta_{x x y}$, and $\delta_{x y y}$. The operators $\delta_{x}$ and $D_{x}$ yield the approximations of first order derivative with respect to $x$, i.e. $\partial_{x}$, and the operators $\delta_{y}$ and $D_{y}$ yield those with respect to $y$, i.e. $\partial_{y}$. The operators $\delta_{x x}, \delta_{y y}, \delta_{x x y}$, and $\delta_{x y y}$ yield the approximations of $\partial_{x x}, \partial_{y y}, \partial_{x x y}$, and $\partial_{x y y}$, respectively. The operators $\delta_{x}$ and $\delta_{x x}$ employ the three point stencil $(i-1, i, i+1)$ and $D_{x}$ employs the five point stencil $(i-2, i-1, i, i+1, i+2)$ for $x=i \epsilon$; the stencil of $\delta_{y}$ and $\delta_{y y}$ is $(j-1, j, j+1)$ and that of $D_{y}$ is $(j-2, j-$ $1, j, j+1, j+2)$ for $y=j \epsilon$. The operator $\delta_{x x y}$ is defined by the product of $\delta_{x x}$ and $\delta_{y}$ and $\delta_{x y y}$ is done by that of $\delta_{x}$ and $\delta_{y y}$. These operators employ the $3 \times 3$ point 
stencil in $x y$ plane $([i-1, i, i+1] \times[j-1, j, j+1])$. The operators $\delta_{x}, \delta_{y}, \delta_{x y y}$, and $\delta_{x x y}$ are second order accurate and the operators $D_{x}, D_{y}, D_{x x}$, and $D_{y y}$ are fourth order accurate. The explicit definitions of these operators are given in Appendix, where the definitions of one-sided finite difference operators, such as $\dot{D}_{x}, \grave{D}_{y}$, and so on, are also shown for later use. The notation of the discretized data for $h=(u, v, P)$ is as follows. $h_{i j}^{n}(h=u, v, P)$ denotes $h\left(n \Delta t, x^{(i)}, y^{(j)}\right)$ and $\delta_{x} h_{i j}^{n}$ and $D_{x} h_{i j}^{n}$ mean the approximations of $\partial_{x} h\left(n \Delta t, x^{(i)}, y^{(j)}\right)$. The other finite difference approximations of derivatives, such as $\partial_{x x} h\left(n \Delta t, x^{(i)}, y^{(j)}\right)$, are denoted in the same way.

Our prototype finite difference scheme consists of

$$
\begin{gathered}
u_{i j}^{n+1 / 2}=u_{i j}^{n}+\frac{\Delta t}{2}\left(-D_{x} P_{i j}^{n}+\nu\left[D_{x x}+D_{y y}\right] u_{i j}^{n}\right), \\
v_{i j}^{n+1 / 2}=v_{i j}^{n}+\frac{\Delta t}{2}\left(-D_{y} P_{i j}^{n}+\nu\left[D_{x x}+D_{y y}\right] v_{i j}^{n}\right), \\
P_{i j}^{n+1 / 2}=P_{i j}^{n}-c^{2} \frac{\Delta t}{2} \mathcal{D}\left(u_{i j}^{n+1 / 2}, v_{i j}^{n+1 / 2}\right), \\
u_{i j}^{n+1}=u_{i j}^{n}+\Delta t\left(-D_{x} P_{i j}^{n+1 / 2}+\nu\left[D_{x x} u_{i j}^{n+1 / 2}+D_{y y} u_{i j}^{n+1 / 2}\right]\right), \\
v_{i j}^{n+1}=v_{i j}^{n}+\Delta t\left(-D_{y} P_{i j}^{n+1 / 2}+\nu\left[D_{x x} v_{i j}^{n+1 / 2}+D_{y y} v_{i j}^{n+1 / 2}\right]\right), \\
P_{i j}^{n+1}=P_{i j}^{n}-c^{2} \Delta t \mathcal{D}\left(u_{i j}^{n+1 / 2}, v_{i j}^{n+1 / 2}\right),
\end{gathered}
$$

where

$$
\mathcal{D}\left(u_{i j}^{n+1 / 2}, v_{i j}^{n+1 / 2}\right)=\delta_{x} u_{i j}^{n+1 / 2}+\delta_{y} v_{i j}^{n+1 / 2}+\frac{\epsilon^{2}}{6}\left(\delta_{x x y} v_{i j}^{n+1 / 2}+\delta_{x y y} u_{i j}^{n+1 / 2}\right) .
$$

The remarks on the above scheme are as follows.

i) $u_{i j}^{n+1 / 2}, v_{i j}^{n+1 / 2}$, and $P_{i j}^{n+1 / 2}$ are the approximation of $u, v$, and $P$ at $(t, x, y)=$ $\left((n+1 / 2) \Delta t, x^{(i)}, y^{(j)}\right)$, respectively.

ii) The operator $\mathcal{D}$, which employs $3 \times 3$ point stencil, gives the approximation of the divergence of the flow velocity $\partial_{x} u+\partial_{y} v$ with the error of $O\left(\epsilon^{2}\right)$. Its accuracy becomes $O\left(\epsilon^{4}\right)$ in the case of the diffusive mode, i.e. $\partial_{x} u+\partial_{y} v \sim$ $O\left(\epsilon^{2}\right)$, since the leading error of $\delta_{x} h\left[\delta_{y} h\right]$ is $\left(\epsilon^{3} / 6\right) \partial_{x x x} h\left[\left(\epsilon^{3} / 6\right) \partial_{y y y} h\right]$ (see Appendix) and the following relations hold:

$$
\begin{aligned}
& \frac{\partial^{3} u}{\partial x^{3}}=-\frac{\partial^{3} v}{\partial x^{2} \partial y}+O\left(\epsilon^{2}\right), \\
& \frac{\partial^{3} v}{\partial y^{3}}=-\frac{\partial^{3} u}{\partial x \partial y^{2}}+O\left(\epsilon^{2}\right) .
\end{aligned}
$$

Owing to the operator $\mathcal{D}$, the stencil for the computation of $P_{i j}^{n+1 / 2}$ and $P_{i j}^{n+1}$ becomes compact. 
iii) The semi-implicit formula is employed in Eq. (62). If $u_{i j}^{n+1 / 1}$ and $v_{i j}^{n+1 / 2}$ in Eq. (62) are replaced by $u_{i j}^{n}$ and $v_{i j}^{n}$, respectively, the scheme becomes unconditionally unstable. The stability of the scheme will be studied in the next subsection.

\subsection{Stability and checkerboard instability}

We examine the stability of the scheme (60)-(65) in the case of $c^{2}=1 /\left(\beta \epsilon^{2}\right)$ by assuming the numerical solution in the form:

$$
\left(\begin{array}{c}
u_{l m}^{n} \\
v_{l m}^{n} \\
P_{l m}^{n}
\end{array}\right)=\lambda^{n} \exp [\mathrm{ik} \epsilon(l+m)]\left(\begin{array}{c}
u_{l m}^{0} \\
v_{l m}^{0} \\
P_{l m}^{0}
\end{array}\right)
$$

where $\lambda, \mathrm{k}$, and $\mathrm{i}$ are the amplification factor, the wave number, and the imaginary number unit $(i=\sqrt{-1})$, respectively. Substituting Eq. (69) into Eqs. (60)(65), we have the characteristic equation, which is a cubic equation for $\lambda$. The roots of the characteristic equation are expressed in the form:

$$
\lambda_{0}=C_{0}(a, b, \nu), \quad \lambda_{ \pm 1}=C_{1}\left(a, b, \nu, \beta^{1 / 2}\right) \pm \sqrt{C_{2}\left(a, b, \nu, \beta^{1 / 2}\right)},
$$

where $a=\cos (\mathrm{k} \epsilon), b=\Delta t / \epsilon^{2}$, and $C_{i}(i=0,1,2)$ are polynomials of the arguments; the expressions of $C_{i}$ are lengthy and are omitted here. The scheme is judged to be stable in the meaning of Von Neumann if $\left|\lambda_{i}\right| \leq 1(i=-1,0,1)$ for $-1 \leq a \leq 1$. From the condition $\left|\lambda_{0}\right| \leq 1$, we have the diffusive CFL condition

$$
b \nu \leq \frac{3}{16},
$$

and it becomes dominant for large $\nu$. From $\left|\lambda_{ \pm 1}\right| \leq 1$, we have the acoustic CFL condition. The formula of the acoustic CFL condition is lengthy and we show it only for the limiting case of $\nu=0$ :

$$
b \leq \frac{9 \beta^{1 / 2}}{\sqrt{19 \sqrt{19}-28}} \sim 1.21556 \beta^{1 / 2} .
$$

The stability range extends as $\beta$ increases, which corresponds to the decrease of sound speed. The acoustic CFL condition is subject to the influence of $\nu$ and the stability range shrinks as $\nu$ increases. For example, the scheme is judged to be stable up to $b \sim 1$ for $(\nu, \beta)=(0.1,1), b \simeq 0.4$ for $(\nu, \beta)=(0.4,1), b \simeq 1.8$ for $(\nu, \beta)=(0.1,4)$, and $b \simeq 0.4$ for $(\nu, \beta)=(0.4,4)$. In the case of $\nu=0.4$, the stability range does not extend even if $\beta$ increases because of the diffusive CFL condition (71).

In Sec. 2.6, we introduced the dashpot-type damping term into the artificial continuity equation. If Eq. (57) is replaced by

$$
\frac{\partial P}{\partial t}+\gamma P+c^{2}\left(\frac{\partial u}{\partial x}+\frac{\partial v}{\partial y}\right)=0
$$


the finite difference formulas (62) and (65) become

$$
\begin{aligned}
P_{i j}^{n+1 / 2} & =\left(P_{i j}^{n}-c^{2} \frac{\Delta t}{2} \mathcal{D}\left(u_{i j}^{n+1 / 2}, v_{i j}^{n+1 / 2}\right)\right) /\left(1+\frac{\gamma \Delta t}{2}\right), \\
P_{i j}^{n+1} & =P_{i j}^{n}-\Delta t\left(\gamma P_{i j}^{n+1 / 2}+c^{2} \mathcal{D}\left(u_{i j}^{n+1 / 2}, v_{i j}^{n+1 / 2}\right)\right) .
\end{aligned}
$$

The change of $\lambda_{ \pm 1}$ by this modification is $O\left(\gamma \epsilon^{2}\right)$ and $\lambda_{0}$ is not altered; the stability range is nearly independent of $\gamma$ while it is $O(1)$ and $\epsilon$ is small.

Since the collocated arrangement of $(u, v, P)$ on the grid is adopted in the present numerical scheme, the checkerboard instability, which is usually observed in the pressure distribution, may occur under a certain computational condition. We will see that this unfavorable numerical phenomenon can be cured by adding another dissipative term to Eq. (75):

$$
P_{i j}^{n+1}=P_{i j}^{n}-\Delta t\left(\gamma P_{i j}^{n+1 / 2}+c^{2} \mathcal{D}\left(u_{i j}^{n+1 / 2}, v_{i j}^{n+1 / 2}\right)-\kappa\left(\delta_{x x}+\delta_{y y}\right) P_{i j}^{n+1 / 2}\right),
$$

where $\kappa$ is a positive constant. The stability range is not altered greatly by the above modification while $\kappa$ is small. For example, the scheme is judged to be stable for $(0 \leq \kappa \lesssim 0.25, b=1,0 \leq \nu \leq 0.1,1 \leq \beta)$. The wave number corresponding to the checkerboard pattern is $\mathrm{k}=\pi / \epsilon(a=-1)$ and the corresponding eigenvalues $\lambda_{i}$ are expressed as

$$
\lambda_{0}=\lambda_{1}=E_{a}, \quad \lambda_{-1}=E_{b}
$$

where

$$
E_{a}=\frac{512(b \nu)^{2}-96 b \nu+9}{9}, \quad E_{b}=\frac{2-b \gamma \epsilon^{2}-16 b \kappa}{2+b \gamma \epsilon^{2}} .
$$

$E_{a}$ is independent of $\gamma$ and $\kappa$ and satisfies $0 \leq E_{a} \leq 1$ under the diffusive CFL condition (71) and the corresponding eigenvectors are $\left(P_{0}, u_{0}, v_{0}\right)=(0,1,0)$, $(0,0,1)$; this mode does not contribute to the pressure variation. $E_{b}$ is independent of $\nu$ and the corresponding eigenvector is $\left(P_{0}, u_{0}, v_{0}\right)=(1,0,0)$; this mode is in charge of the checkerboard pattern for the pressure. In the case of the original prototype scheme $(\gamma=\kappa=0)$, i.e. $E_{b}=1$, it is concluded that the checkerboard mode is not suppressed. The contribution of the parameter $\gamma$ to the suppression of checkerboard instability is $O\left(\epsilon^{2}\right)$ and the effective contribution is given by the parameter $\kappa$. Unless $\kappa \lesssim \epsilon^{2}$, however, the second order accuracy of the time-integration is spoiled. Fortunately, the side effect of the cure will be eliminated in the actual finite-difference scheme of ACE by making use of the property of the diffusive mode (Sec. 4).

\section{FINITE DIFFERENCE SCHEME OF ACE}

We will extend the prototype scheme in the previous section to the case of ACE. The treatment of the boundary condition will be the main topic. Similar to the prototype scheme, the explanation will be given for $2 \mathrm{D}$ case. 
$\mathrm{ACE}(2)$ and (41) in 2D case is rewritten as

$$
\begin{gathered}
\frac{\partial P}{\partial t}+\gamma P+\frac{1}{\beta \epsilon^{2}}\left(\frac{\partial u}{\partial x}+\frac{\partial v}{\partial y}\right)=0, \\
\frac{\partial u}{\partial t}+u \frac{\partial u}{\partial x}+v \frac{\partial u}{\partial y}+\frac{\partial P}{\partial x}=\nu\left(\frac{\partial^{2} u}{\partial x^{2}}+\frac{\partial^{2} u}{\partial y^{2}}\right)+F, \\
\frac{\partial v}{\partial t}+u \frac{\partial v}{\partial x}+v \frac{\partial v}{\partial y}+\frac{\partial P}{\partial y}=\nu\left(\frac{\partial^{2} v}{\partial x^{2}}+\frac{\partial^{2} v}{\partial y^{2}}\right)+G,
\end{gathered}
$$

where $(F, G)$ is the external force. For the simple explanation, we consider the problem for the case where the numerical domain $\Omega$ is the rectangle $[0 \leq x \leq$ $\left.L_{x}\right] \times\left[0 \leq y \leq L_{y}\right]$. Then, its boundary $\partial \Omega$ consists of four line segments defined by $\Gamma_{x 0}=\left(x=0,0 \leq y \leq L_{y}\right), \Gamma_{x 1}=\left(x=L_{x}, 0 \leq y \leq L_{y}\right), \Gamma_{y 0}=(y=$ $\left.0,0 \leq x \leq L_{x}\right)$, and $\Gamma_{y 1}=\left(y=L_{y}, 0 \leq x \leq L_{x}\right)$. We employ the grid system with the spacing $\epsilon$ : $\left(x^{(i)}, y^{(j)}\right)=(i \epsilon, j \epsilon)\left(i=0,1,2, \cdots, N_{x} ; j=0,1,2, \cdots, N_{y}\right.$; $\left.N_{x} \epsilon=L_{x} ; N_{y} \epsilon=L_{y}\right)$.

\subsection{Boundary value of pressure}

The finite difference formulas for the grid points in the vicinity of the boundary require the boundary values of $(u, v, P)$. While the boundary data for $u$ and $v$ are supplied from the boundary condition, no explicit data is given for $P$. Since the finite difference approximation of the pressure derivatives in the momentum equation must be fourth order accurate, the boundary data of the pressure must be at least fifth order accurate (the boundary value of pressure is divided by $\epsilon$ in the finite-difference approximation of pressure gradient; see Appendix). In this subsection, we will explain the computation of $P$ at the boundary $\Gamma_{y 0}(y=0)$. The computation for the other boundary segments can be done in the same way and the explanation is omitted. Since the following procedure is independent of $t$, we will express $h(t, x, y)(h=u, v, P)$ as $h(x, y)$ below.

The boundary value $P(x, 0)$ is computed by using the one-sided finite difference formula

$$
P_{0}=\left[48 P_{1}-36 P_{2}+16 P_{3}-3 P_{4}-12 \epsilon \frac{\partial P}{\partial y}(x, 0)\right] / 25+O\left(\epsilon^{5}\right),
$$

where $P_{j}$ stands for $P(x, j \epsilon)$. The value of $\partial P / \partial y(x, 0)$ in the above formula is supplied via the momentum equation for $v$ [Eq. (81)]. The values of $u, v, \partial v / \partial x$, $\partial^{2} v / \partial x^{2}$, and $\partial v / \partial t$ can easily be computed by using the boundary condition and the problem is reduced to the computation of $\partial v / \partial y$ and $\partial^{2} v / \partial y^{2}$ at $y=$ 0 . The derivative $\partial v / \partial y(x, 0)$ is computed by the one-sided finite difference formula:

$$
\frac{\partial v}{\partial y}(x, 0)=\frac{-25 v_{0}+48 v_{1}-36 v_{2}+16 v_{3}-3 v_{4}}{12 \epsilon}+O\left(\epsilon^{4}\right)
$$


where $v_{j}$ stands for $v(x, j \epsilon)$. The second derivative $\partial^{2} v / \partial y^{2}(x, 0)$ is given by

$$
\frac{\partial^{2} v}{\partial y^{2}}(x, y=0)=\frac{35 v_{0}-104 v_{1}+114 v_{2}-56 v_{3}+11 v_{4}}{12 \epsilon^{2}}-\frac{5 \epsilon^{3}}{6} \frac{\partial^{5} v}{\partial y^{5}}(x, 0)+O\left(\epsilon^{4}\right) .
$$

We have, for the diffusive mode,

$$
\frac{\partial^{5} v}{\partial y^{5}}=-\frac{\partial^{5} u}{\partial x \partial y^{4}}+O\left(\epsilon^{2}\right)
$$

Further, we have

$$
\begin{gathered}
\frac{\partial^{5} u}{\partial x \partial y^{4}}(x, y=0)=\frac{H(x+\epsilon)-H(x-\epsilon)}{2 \epsilon^{5}}+O(\epsilon), \\
H(x)=u_{0}-4 u_{1}+6 u_{2}-4 u_{3}+u_{4},
\end{gathered}
$$

where $u_{j}(j=0,1,2,3,4$ are the abbreviations of $u(x, j \epsilon)$. This completes the fifth order accurate formula of $P(x, 0)$.

\subsection{Finite difference formulas for inner grid points}

The finite difference formulas for the inner grid points $\left(2 \leq i \leq N_{x}-2,2 \leq\right.$ $\left.j \leq N_{y}-2\right)$ are given by

$$
\begin{gathered}
u_{i j}^{n+1 / 2}=u_{i j}^{n}+\frac{\Delta t}{2}\left(-u_{i j}^{n} D_{x} u_{i j}^{n}-v_{i j}^{n} D_{y} u_{i j}^{n}-D_{x} P_{i j}^{n}+\nu\left[D_{x x}+D_{y y}\right] u_{i j}^{n}+F_{i j}^{n}\right), \\
v_{i j}^{n+1 / 2}=v_{i j}^{n}+\frac{\Delta t}{2}\left(-u_{i j}^{n} D_{x} v_{i j}^{n}-v_{i j}^{n} D_{y} v_{i j}^{n}-D_{y} P_{i j}^{n}+\nu\left[D_{x x}+D_{y y}\right] v_{i j}^{n}+G_{i j}^{n}\right),(89) \\
P_{i j}^{n+1 / 2}=\left(P_{i j}^{n}-\frac{\Delta t}{2 \beta \epsilon^{2}} \mathcal{D}\left(u_{i j}^{n+1 / 2}, v_{i j}^{n+1 / 2}\right)\right) /\left(1+\frac{\gamma \Delta t}{2}\right), \\
u_{i j}^{n+1}=u_{i j}^{n}+\Delta t\left(-u_{i j}^{n+1 / 2} D_{x} u_{i j}^{n+1 / 2}-v_{i j}^{n+1 / 2} D_{y} u_{i j}^{n+1 / 2}\right. \\
\left.\quad-D_{x} P_{i j}^{n+1 / 2}+\nu\left[D_{x x}+D_{y y}\right] u_{i j}^{n+1 / 2}+F_{i j}^{n+1 / 2}\right), \\
v_{i j}^{n+1}=v_{i j}^{n}+\Delta t\left(-u_{i j}^{n+1 / 2} D_{x} v_{i j}^{n+1 / 2}-v_{i j}^{n+1 / 2} D_{y} v_{i j}^{n+1 / 2}\right. \\
\left.\quad-D_{y} P_{i j}^{n+1 / 2}+\nu\left[D_{x x}+D_{y y}\right] v_{i j}^{n+1 / 2}+G_{i j}^{n+1 / 2}\right), \\
P_{i j}^{n+1}=P_{i j}^{n}+\Delta t\left(-\gamma P_{i j}^{n+1 / 2}-\frac{1}{\beta \epsilon^{2}} \mathcal{D}\left(u_{i j}^{n+1 / 2}, v_{i j}^{n+1 / 2}\right)\right. \\
\left.+\mu \epsilon\left[\left(\delta_{x x}+\delta_{y y}\right) P_{i j}^{n+1 / 2}+2\left(\delta_{x} v_{i j}^{n+1 / 2} \delta_{y} u_{i j}^{n+1 / 2}-\delta_{x} u_{i j}^{n+1 / 2} \delta_{y} v_{i j}^{n+1 / 2}\right)\right]\right),
\end{gathered}
$$

where $F_{i j}^{s}=F\left(s \Delta t, x^{(i)}, y^{(j)}\right)$ and $G_{i j}^{s}=G\left(s \Delta t, x^{(i)}, y^{(j)}\right)(s=n, n+1 / 2)$ and $\mu$ is a positive constant of the order of unity.

The remarks on the above formulas are as follows. 
i) The nonlinear terms and the external force are added in Eqs. (88), (89), (91), and (92).

ii) The term

$$
\mu \epsilon\left[\left(\delta_{x x}+\delta_{y y}\right) P_{i j}^{n+1 / 2}+2\left(\delta_{x} v_{i j}^{n+1 / 2} \delta_{y} u_{i j}^{n+1 / 2}-\delta_{x} u_{i j}^{n+1 / 2} \delta_{y} v_{i j}^{n+1 / 2}\right)\right]
$$

in Eq. (93) corresponds to $\kappa\left(\delta_{x x}+\delta_{y y}\right) P_{i j}^{n+1 / 2}$ in Eq. (76), which is introduced as the cure for checkerboard instability. It becomes $O\left(\epsilon^{3}\right)$ in the case of the diffusive mode. This is seen by noting

$$
\frac{\partial^{2} P}{\partial x^{2}}+\frac{\partial^{2} P}{\partial y^{2}}+2\left(\frac{\partial u}{\partial y} \frac{\partial v}{\partial x}-\frac{\partial u}{\partial x} \frac{\partial v}{\partial y}\right)=O\left(\epsilon^{2}\right)
$$

which is derived from Eqs. (41) and (2) with $k=\beta \epsilon^{2}$. Therefore, the second order accuracy with respect to time is not spoiled in the case of the diffusive mode.

\subsection{Computation at grid points next to boundary}

We will explain the computation at the grid points next to the boundary, i.e. $(i, 1)$ and $\left(i, N_{y}-1\right)$ for $1 \leq i \leq N_{x}-1$ and $(1, j)$ and $\left(N_{x}-1, j\right)$ for $2 \leq j \leq N_{y}-2$. In the following explanation, we will use various finite difference operators, the definitions of which are given in Appendix.

Since the stencil of the pressure update, Eqs. (90) and (93), is compact, no modification is necessary in this case. However, the velocity update, i.e. Eqs. (88), (89), (91), and (92), employs the five point central finite difference operators $D_{x}$ and $D_{y}$ and at least one of them is not available at the grid points next to the boundary. We can employ the one-sided five point finite difference operators $\dot{D}_{x}$ or $\grave{D}_{x}$ instead of $D_{x}$ and $\dot{D}_{y}$ or $\grave{D}_{y}$ instead of $D_{y}$. Then, the fourth order accuracy is assured for the convective terms and the pressure gradient. As for the second derivatives in the viscous terms, e.g. $\partial_{x x} u$ and $\partial_{y y} v$, the central five point finite difference operators $D_{x x}$ and $D_{y y}$ are not available and the one-sided 5 point finite difference operators $\dot{D}_{x x}, \grave{D}_{y y}, \grave{D}_{x x}$, and $\grave{D}_{y y}$ are third order accurate. A larger stencil is required for the realization of the fourth order accuracy but it is not advantageous for the stability. Here, we propose an alternative method based on the well-known technique, called Numerov algorithm. For the concise expressions of formulas, we express $\delta_{x} u, \delta_{y} v, \delta_{x y y} P$, $\delta_{x x} \delta_{y y} u$ as $U_{x}, V_{y}, P_{x y y}$, and $U_{x x y y}$, respectively; $U_{y y}, V_{x x}, U_{x y y}$, and so on, are defined in the same way. The five point finite difference approximations $D_{x} u$, $D_{y} v, D_{x x} u$, and so on, will be expressed as they are.

In Numerov algorithm, the momentum equations (80) and (81) are modified. In the case of the grids points $(i, 1)(2 \leq i \leq N-2)$, which are next to $\Gamma_{y 0}$, the derivation is as follows. We multiply both hand sides of Eq. (80) by $1+$ $\left(\epsilon^{2} / 12\right) \delta_{y y}$. Noting

$$
1+\frac{\epsilon^{2}}{12} \delta_{y y}=1+\frac{\epsilon^{2}}{12} \frac{\partial^{2}}{\partial y^{2}}+O\left(\epsilon^{4}\right)
$$


and Eq. (120) in Appendix, we have

$$
\begin{aligned}
\frac{\partial \tilde{u}}{\partial t}= & -u D_{x} u-v \dot{D}_{y} u-D_{x} P \\
& -\frac{\epsilon^{2}}{12}\left(U_{y y} U_{x}+2 U_{y} U_{x y}+u U_{x y y}+V_{y y} U_{y}+2 V_{y} U_{y y}+v \dot{D}_{y y y} u+P_{x y y}\right) \\
& +\nu\left(D_{x x} u+U_{y y}+\frac{\epsilon^{2}}{12} U_{x x y y}\right)+\tilde{F}+O\left(\epsilon^{4}\right),
\end{aligned}
$$

where

$$
\begin{gathered}
\tilde{u}=\left(1+\frac{\epsilon^{2}}{12} \delta_{y y}\right) u, \\
\tilde{F}=\left(1+\frac{\epsilon^{2}}{12} \delta_{y y}\right) F .
\end{gathered}
$$

Similarly, we have from Eq. (81)

$$
\begin{aligned}
\frac{\partial \tilde{v}}{\partial t}= & -u D_{x} v-v \dot{D}_{y} v-\dot{D}_{y} P \\
& -\frac{\epsilon^{2}}{12}\left(U_{y y} V_{x}+2 U_{y} V_{x y}+u V_{x y y}+3 V_{y y} V_{y}+v \dot{D}_{y y y} v+\dot{D}_{y y y} P\right) \\
& +\nu\left(D_{x x} v+V_{y y}+\frac{\epsilon^{2}}{12} V_{x x y y}\right)+\tilde{G}+O\left(\epsilon^{4}\right),
\end{aligned}
$$

where

$$
\begin{gathered}
\tilde{v}=\left(1+\frac{\epsilon^{2}}{12} \delta_{y y}\right) v, \\
\tilde{G}=\left(1+\frac{\epsilon^{2}}{12} \delta_{y y}\right) G .
\end{gathered}
$$

The modified momentum equation for the grid points $(i, N-1)(2 \leq i \leq N-2)$, which are next to $\Gamma_{y 1}$, is obtained from the above formula by the replacement $\left(\dot{D}_{y}, \dot{D}_{y y y}\right) \rightarrow\left(\grave{D}_{y}, \grave{D}_{y y y}\right)$. The modified momentum equations for the grid points $(1, j)(2 \leq j \leq N-2)$ are given by

$$
\begin{aligned}
\frac{\partial \bar{u}}{\partial t}= & -u \dot{D}_{x} u-v D_{y} u-\dot{D}_{x} P \\
& -\frac{\epsilon^{2}}{12}\left(3 U_{x x} U_{x}+u \dot{D}_{x x x} u+V_{x x} U_{y}+2 V_{x} U_{x y}+v U_{x x y}+\dot{D}_{x x x} P\right) \\
& +\nu\left(U_{x x}+D_{y y} u+\frac{\epsilon^{2}}{12} U_{x x y y}\right)+\bar{F}+O\left(\epsilon^{4}\right), \\
\frac{\partial \bar{v}}{\partial t}= & u \dot{D}_{x} v-v D_{y} v-D_{y} P \\
- & \frac{\epsilon^{2}}{12}\left(U_{x x} V_{x}+2 U_{x} V_{x x}+u \dot{D}_{x x x} v+V_{x x} V_{y}+2 V_{x} V_{x y}+v V_{x x y}+P_{x x y}\right) \\
+ & \nu\left(V_{x x}+D_{y y} v+\frac{\epsilon^{2}}{12} V_{x x y y}\right)+\bar{G}+O\left(\epsilon^{4}\right),
\end{aligned}
$$


where

$$
\begin{array}{ll}
\bar{u}=\left(1+\frac{\epsilon^{2}}{12} \delta_{x x}\right) u, & \bar{F}=\left(1+\frac{\epsilon^{2}}{12} \delta_{x x}\right) F, \\
\bar{v}=\left(1+\frac{\epsilon^{2}}{12} \delta_{x x}\right) v, & \bar{G}=\left(1+\frac{\epsilon^{2}}{12} \delta_{x x}\right) G .
\end{array}
$$

Those for the grid points $(N-1, j)(2 \leq j \leq N-2)$, which are next to $\Gamma_{x 1}$, are obtained from the above formula by the replacement $\left(\dot{D}_{x}, \dot{D}_{x x x}\right) \rightarrow\left(\grave{D}_{x}, \grave{D}_{x x x}\right)$.

At the grid points $(1,1),\left(N_{x}-1,1\right),\left(N_{x}-1, N_{y}-1\right)$, and $\left(1, N_{y}-1\right)$, the finite difference operators $D_{x x}$ and $D_{y y}$ are not available. In this case, Numerov algorithm requires the multiplication of $1+\left(\epsilon^{2} / 12\right)\left(\delta_{x x}+\delta_{y y}\right)$ by both hand sides of Eqs. (80) and (81). Then, we encounter another difficulty in the computation of $\left(\delta_{x x}+\delta_{y y}\right) \partial_{\alpha} P(\alpha=x, y)$. Fortunately, we can compute $\left(\partial_{x}^{2}+\partial_{y}^{2}\right) \partial_{\alpha} P$ as the finite difference approximation of $u$ and $v$ by making use of Eq. (94). We define $\hat{u}$ and $\hat{v}$ :

$$
\begin{aligned}
& \hat{u}=\left(1+\frac{\epsilon^{2}}{12}\left(\delta_{x x}+\delta_{y y}\right)\right) u, \\
& \hat{v}=\left(1+\frac{\epsilon^{2}}{12}\left(\delta_{x x}+\delta_{y y}\right)\right) v .
\end{aligned}
$$

The modified momentum equations for the grid point $(1,1)$ are

$$
\begin{aligned}
\frac{\partial \hat{u}}{\partial t}= & -u \dot{D}_{x} u-v \dot{D}_{y} u-\dot{D}_{x} P \\
& -\frac{\epsilon^{2}}{12}\left[3 U_{x} U_{x x}+u \dot{D}_{x x x} u+V_{x x} U_{y}+2 V_{x} U_{x y}+v U_{x x y}\right. \\
& +U_{x} U_{y y}+2 U_{y} U_{x y}+u U_{x y y}+V_{y y} U_{y}+2 V_{y} U_{y y}+v \dot{D}_{y y y} u \\
& \left.+2\left(U_{x x} V_{y}+U_{x} V_{x y}-U_{x y} V_{x}-U_{y} V_{x x}\right)\right] \\
& +\nu\left(U_{x x}+V_{y y}+\frac{\epsilon^{2}}{6} U_{x x y y}\right)+\hat{F} \\
\frac{\partial \hat{v}}{\partial t}= & -u \dot{D}_{x} v-v \dot{D}_{y} v-\dot{D}_{y} P \\
& -\frac{\epsilon^{2}}{12}\left[V_{x} U_{x x}+2 U_{x} V_{x x}+u \dot{D}_{x x x} v+V_{x x} V_{y}+2 V_{x} V_{x y}+v V_{x x y}\right. \\
& +V_{x} U_{y y}+2 U_{y} V_{x y}+u V_{x y y}+3 V_{y} V_{y y}+v \dot{D}_{y y y} v \\
& \left.+2\left(U_{x y} V_{y}+U_{x} V_{y y}-U_{y y} V_{x}-U_{y} V_{x y}\right)\right] \\
& +\nu\left(V_{x x}+V_{y y}+\frac{\epsilon^{2}}{6} V_{x x y y}\right)+\hat{G}
\end{aligned}
$$

where

$$
\hat{F}=\left(1+\frac{\epsilon^{2}}{12}\left(\delta_{x x}+\delta_{y y}\right)\right) F, \quad \hat{G}=\left(1+\frac{\epsilon^{2}}{12}\left(\delta_{x x}+\delta_{y y}\right)\right) G .
$$


Those for the other three grid points are obtained by the appropriate replacement of one-sided finite-difference operators, e.g. $\left(\dot{D}_{x}, \dot{D}_{x x x}\right) \rightarrow\left(\grave{D}_{x}, \grave{D}_{x x x}\right)$ at the grid point $(N-1, N-1)$.

As the example of computation of Numerov algorithm, we explain the computation procedure for $(i, j)=(i, 1)\left(1<i<N_{x}\right)$ below. Let $\hat{u}$ and $\hat{v}$ at $(x, y, t)=(i \epsilon, j \epsilon, n \Delta t)$ be denoted by $\hat{u}_{i j}^{n}$ and $\hat{v}_{i j}^{n}$, respectively.

i) Compute $\tilde{u}_{i, 1}^{n}$ from $u_{i, 0}^{n}, u_{i, 1}^{n}, u_{i, 2}^{n}$ by using Eq. (97). Compute $\tilde{v}_{1,1}^{n}$ by using Eq. (100) in the similar way.

ii) Compute $\tilde{u}_{i, 1}^{n+1 / 2}$ and $\tilde{v}_{i, 1}^{n+1 / 2}$ by using the first order accurate time integration method (Euler method) for Eqs. (96) and (99), respectively.

iii) Compute $u_{i, 1}^{n+1 / 2}$ from $\tilde{u}_{i, 1}, u_{i, 2}^{n+1 / 2}$, and $u_{i, 0}^{n+1 / 2}$ according to Eq. (97), i.e.

$$
u_{i, 1}^{n+1 / 2}=\frac{6}{5} \tilde{u}_{i, 1}^{n+1 / 2}-\frac{5}{2}\left(u_{i, 2}^{n+1 / 2}+u_{i, 0}^{n+1 / 2}\right),
$$

where $u_{i, 2}^{n+1 / 2}$ is computed by using Eq. (88) and $u_{i, 0}^{n+1 / 2}$ is given by the boundary condition. Carry out the inverse transformation $\tilde{v}_{i, 1}^{n+1 / 2} \rightarrow$ $v_{i, 1}^{n+1 / 2}$ in the similar way.

iv) Compute $\tilde{u}_{i, 1}^{n+1}$ and $\tilde{v}_{i, 1}^{n+1}$ by using the midpoint rule for Eqs. (96) and (99), respectively.

v) Carry out the inverse transformation $\left(\tilde{u}_{i, 1}^{n+1}, \tilde{v}_{i, 1}^{n+1}\right) \rightarrow\left(u_{i, 1}^{n+1}, v_{i, 1}^{n+1}\right)$ in the same way as step iii).

The computation for $\left(i, N_{y}-1\right)$ for $\left(2 \leq i \leq N_{x}-2\right)$ and $(1, j)$ and $\left(N_{x}-1, j\right)$ $\left(2 \leq j \leq N_{y}-2\right)$ can be done in the same way. Finally, as an example of the computation for four grid points $(1,1),\left(1, N_{x}-1\right),\left(N_{x}-1, N_{y}-1\right)$, and $\left(1, N_{y}-1\right)$, we show the formula of inverse transformation for obtaining $u_{1,1}^{n+1 / 2}$ :

$$
u_{1,1}^{n+1 / 2}=\frac{3}{2} \hat{u}_{1,1}^{n+1 / 2}-\frac{1}{8}\left(u_{1,2}^{n+1 / 2}+u_{1,0}^{n+1 / 2}+u_{0,1}^{n+1 / 2}+u_{2,1}^{n+1 / 2}\right),
$$

where $u_{1,0}^{n+1 / 2}$ and $u_{0,1}^{n+1 / 2}$ are given by the boundary condition and $u_{2,1}^{n+1 / 2}$ and $u_{1,2}^{n+1 / 2}$ are computed in advance by the inverse transformation of $\tilde{u}_{2,1}^{n+1 / 2}$ and $\bar{u}_{1,2}^{n+1 / 2}$.

\section{NUMERICAL TESTS}

\subsection{Generalized Taylor-Green vortex problem}

The problem of Taylor-Green vortices is widely employed as a test case for INSE solvers because of the availability of its simple analytical solution. The solution of the original problem decays exponentially as time advances because 
of viscous dissipation. In this subsection, we demonstrate the decay of acoustic mode besides the convergence of numerical solution. In order to avoid the confusion between the decay of solution and that of acoustic mode, we modify the original problem such that the solution is periodic with respect to time as well. By applying the external force

$$
\begin{aligned}
& F(t, x, y)=\sin \left(x-u_{0} t\right) \cos \left(y-v_{0} t\right)[2 \nu \cos t-\sin t] \\
& G(t, x, y)=-\cos \left(x-u_{0} t\right) \sin \left(y-v_{0} t\right)[2 \nu \cos t-\sin t]
\end{aligned}
$$

to the momentum equations, we have the exact solution of INSE

$$
\begin{aligned}
& u(t, x, y)=u_{0}+\sin \left(x-u_{0} t\right) \cos \left(y-v_{0} t\right) \cos t, \\
& v(t, x, y)=v_{0}-\cos \left(x-u_{0} t\right) \sin \left(y-v_{0} t\right) \cos t, \\
& P(t, x, y)=\frac{1}{4}\left[\cos 2\left(x-u_{0} t\right)+\cos 2\left(y-v_{0} t\right)\right] \cos ^{2} t,
\end{aligned}
$$

where $u_{0}$ and $v_{0}$ are constants and they are introduced in order to avoid the special situation where the advection term balances with the pressure gradient. We solve the above modified problem numerically in the domain $\Omega=[0 \leq x \leq$ $2 \pi] \times[0 \leq y \leq 2 \pi]$ with the periodic boundary condition. Incidentally, INSE is Galilean invariant but ACE is not. Therefore, the error of ACM depends on the values of $u_{0}$ and $v_{0}$.

During the computation, we measured the error defined by

$$
E[h](t)=\frac{1}{4 \pi^{2}} \int_{0}^{2 \pi} \int_{0}^{2 \pi}\left|h_{N u m}(x, y, t)-h_{\text {Exact }}(x, y, t)\right| d x d y,
$$

where $h_{N u m}$ and $h_{\text {Exact }}(h=u, v, P)$ stand for the numerical solution and the exact solution, respectively, and the integration is evaluated numerically. The time history of $E[h](h=u, v, P)$ for $\nu=0.001$ and $\left(u_{0}, v_{0}\right)=(0.3,0.6)$ $\left[\epsilon=\pi / 128\left(N_{x}=N_{y}=256\right), \Delta t=\epsilon^{2}(b=1), \beta=2\right.$ and $\left.\beta=4, \gamma=\mu=1\right]$ is shown in Figs. 1 and 2. The diffusive mode, the time scale of which is $O(1)$, and the acoustic mode, that of which is $O(\epsilon)$, are seen in these figures. As expected from the discussion in Sec 2, the magnitude of acoustic mode increases and its frequency decreases as $\beta$ increases (Fig. 1). In Fig. 2, the curves for $\beta=2$ overlaps with that for $\beta=4$ after the extinction of the acoustic mode. Since the scale for $E[h(\beta=4])(h=u, v, P)$ is doubled, the overlap of two curves implies the linear dependence of $E[h]$ on $\beta$. The data of $E[h(\beta=2)], E[h(\beta=4)$, and $E[2 h(\beta=2)-h(\beta=4)]$ at $t=60(\nu=0.001, \gamma=\mu=b=1)$ for different resolutions are shown in Table 1 . It is seen from the table that the error is drastically reduced by combining two solutions for different values of $\beta$. While the convergence rate for $\beta=2$ and $\beta=4$ are almost second order in $\epsilon$, that for the linear combination of the two numerical solutions, which will hereafter be called the refined solution, is nearly fourth order. We should remark that the clear demonstration of drastic error reduction becomes possible only when the acoustic mode is suppressed sufficiently. In fact, in the case of $\gamma=0$, the magnitude of the acoustic mode at $t=60$ is estimated from the time history of 
error to be of the same order as that of the data in the table. The computation using the second order accurate scheme, the formulas of which are derived by replacing the five point finite difference operators $D_{x}, D_{y}, D_{x x}$ and $D_{y y}$ in Eqs. (88), (89), (91), and (92) by three point finite difference operators $\delta_{x}, \delta_{y}$, $\delta_{x x}$, and $\delta_{y y}$, respectively; the stencil of 2 nd order accurate scheme is the same as D2Q9 of LBM. The data corresponding to the case of Table-1 are shown in Table-2. The data for $E[h(\beta=2)]$ and $E[h(\beta=4)]$ show nearly 2 nd order convergence rate. Although the error is reduced by combining two solutions as before, it is much larger than that of refined solution shown in Table- 1 and the convergence rate is still second order.

The error data for the case of $\nu=0.1(\gamma=\mu=b=1)$ are shown in Table3 (fourth order accurate scheme) and Table-4 (second order accurate scheme). Although the convergence rate of the refined solution is not fourth order but nearly third order, the error is drastically reduced (Table-3). In Table-4, the error data of MRT-LBM [5, 11] with the consistent treatment of forcing of Ref. [4] are shown for comparison. The time step employed in the LBM computation is the same as that of ACM, i.e. $\Delta t=\epsilon^{2}$, and the tuning parameters of MRT are $s_{1}=s_{4}=s_{6}\left(=\tau_{\rho}^{-1}=\tau_{j}^{-1}\right)=0, s_{2}\left(=\tau_{e}^{-1}\right)=1.63, s_{3}\left(=\tau_{\epsilon}^{-1}\right)=1.14$, $s_{5}=s_{7}\left(=\tau_{q}{ }^{-1}\right)=1.92$ (see Refs. [11, 4]). MRT-LBM with the consistent treatment of forcing is one of the most up-to-date LBMs, however, it produces larger errors than those of simple second order accurate ACM, which employs the same compact stencil. As seen from the clear second order convergence rate for MRT-LBM, this is not due to insufficient decay of acoustic mode, which is also confirmed from the time history of error (no figure). Incidentally, the computation cost of ACM (second order scheme and fourth order one) and that of LBM were nearly the same in the vector and parallel computing environment using OpenMP.

\subsection{Formation of two rolls by moving wall}

Let us consider the IBVP of INSE in the domain $\Omega=[0 \leq x \leq 2 \pi] \times[0 \leq$ $y \leq 2 \pi]$ with the homogeneous initial data

$$
u(t=0)=v(t=0)=P(t=0)=0,
$$

and the boundary condition

$$
\begin{aligned}
& u(t, x, 0)=-u(t, x, 2 \pi)=\frac{1}{2} \sin x \exp \left(-3 / t^{2}\right), \quad v(t, x, 0)=v(t, x, 2 \pi)=0 \\
& v(t, 0, y)=v(t, 2 \pi, y)=-\sin (y / 2) \exp \left(-3 / t^{2}\right), \quad u(t, 0, y)=u(t, 2 \pi)=0 .
\end{aligned}
$$

The wall speeds are designed in such a way that a clockwise roll (right) and a counterclockwise one (left) are formed and the factor $\exp \left(-3 / t^{2}\right)$ is for smooth start. The solution of INSE must exhibit the symmetry with respect to the line $x=\pi ; u$ is odd and $v$ and $P$ are even with respect to the line, i.e. $u(t, \pi+x, y)=$ $-u(t, \pi-x, y), v(t, \pi+x, y)=v(t, \pi-x, y)$, and $P(t, \pi+x, y)=P(t, \pi-x, y)$. 
The flow velocity field for INSE is not altered even if a conservative force is added; it is incorporated into the pressure. ACE, however, does not have this property and the symmetry breaking of flow velocity field appears as the error in the case of asymmetric potential. We will make use of it for the graphical demonstration of error reduction of ACM. In the following numerical example, the external force is $(F, G)=(-0.2,-0.1)$ and the initial pressure field with zero average is modified accordingly, i.e.

$$
P(t=0, x, y)=\frac{-(2 x+y)+3 \pi}{10} .
$$

The numerical computation was done for $\nu=0.01$ and $\nu=0.001(\gamma=\mu=$ $b=1, \beta=2$ and $\beta=4$ ). The refined solution at $t=30$ for $\nu=0.01$ and that for $\nu=0.001(\epsilon=\pi / 512)$ are shown in Figs. 3 and 4, respectively. These figures show the symmetry of streamlines and the asymmetry of the pressure field. The velocity fields for $\beta=2$ and $\beta=4$ and that for the refined solution in the case of $(\nu=0.01, \epsilon=\pi / 64)$ are shown in Fig. 5 and those in the case of $(\nu=0.001$, $\epsilon=\pi / 128)$ are shown in Fig. 6 . The refined solutions for $\epsilon=\pi / 512$ are also shown in these figures as the reference solutions. While the velocity fields for $\beta=2$ and $\beta=4$ display the asymmetry, the refined solutions agree very well with the symmetric reference solutions. Next, we will check the convergence numerically. For this purpose, we generate the reference solution from the two refined solutions for $\epsilon=\pi / 256$ and $\epsilon=\pi / 512$ by making use of Richardson extrapolation under the assumption that the leading error of the refined solution is $O\left(\epsilon^{4}\right)$. The $L^{1}$ errors $E[h](h=u, v, P)$ are computed by Eq. (113) with the replacement of the exact solution by the so generated reference solution. The data of error for $\nu=0.01$ are shown in Table- 5 and those for $\nu=0.001$ are done in Table- 6 . In both cases, the error is reduced greatly by taking the linear combination and the convergence rate is nearly fourth order.

\subsection{Lid-driven cavity flow}

The problem of lid-driven cavity flow is a very popular test case and it deals with the motion of a fluid confined in a square domain $\Omega=[0 \leq x \leq 1] \times[0 \leq$ $y \leq 1]$ consisting of the top side $(y=1)$ with an imposed velocity $(u=U(x)$ and $v=0)$ and the other three sides at rest. No external force is acting on the system $(F=G=0)$. Here, we consider the usual case of $U(x)=1$ with the impulse start from the homogeneous initial data $u=v=P=0$. Because of the discontinuities with respect to space and time, the regularity of solution is obviously lost. Such situations are often encountered in practical applications and the fourth order accuracy is not realized. The scheme should at least prove its ability of stable computation even in such situations, however. Here, we demonstrate the perfomance of the scheme in the steady case.

As for the existing steady solutions for high Reynolds numbers, those for $\nu=0.0002(\operatorname{Re}=5000)$ are found in the literature (see Ref. [2] and the references therein). Since the accuracy with respect to time is not required, it is advantageous to carry out the computation for a large time step by relaxing the severe acoustic CFL condition. In our computation, the value of $\beta$ 
and the time step $\Delta t$ were $\beta \epsilon^{2}=\mathrm{Ma}=0.3$ and $\Delta t=0.24 \epsilon$. The value of $\gamma$ was set to be zero since the acoustic mode dies in the steady state but $\mu$ was set to be unity as before for the cure of checkerboard instability. The $x$ component of flow velocity $u$ has two limiting values at the two top corners $(x, y)=(0,1),(1,1)\left[(i, j)=\left(0, N_{y}\right),\left(N_{x}, N_{y}\right)\right]$. We first carried out the computation setting $u=1$ at these two corners. The computation was done safely without producing any spurious oscillations. The numerical results were compared with the accurate solution of Ref. [2], which is obtained for $2048 \times 2048$ uniform staggered mesh system. While good agreement with the reference solution was confirmed for the overall pressure distribution, the deviation from the reference solution was still appreciable for the velocity even in the case of $\epsilon=1 / 1024\left[\left(N_{x}, N_{y}\right)=(1024,1024)\right]$ (no figure). The mesh system employed in the computation includes the grid points at the boundary and the two top corners with singularity are not excluded from the stencils of the computation of divergence of velocity at the grid points $(i, j)=\left(1, N_{y}-1\right),\left(N_{x}-1, N_{y}-1\right)$ [see Eq. (66) and Appendix]. In order to relax the influence of corner singularity, we modified the code such that the divergence of the flow velocity at these two points is computed by using Gauss's theorem; the divergence of flow velocity at $(i, j)=\left(1, N_{y}-1\right)$ is approximated as the volume average over the four neighboring cells $(0 \leq x \leq 2 \epsilon) \times(1-2 \epsilon \leq y \leq 1)$, which is computed numerically as the line integral of mass flux; the divergence of flow velocity at $(i, j)=\left(N_{x}-1, N_{y}-1\right)$ is computed in the same way. The result of the modified code for $\left(N_{x}, N_{y}\right)=(256,256)$ is shown together with the reference solution in Figs. 7-9. The deviation from the reference solution becomes nearly invisible. However, it should be noted that the divergence of flow velocity is $O(1)$ in the vicinities of the two top corners and it balances with the term of the cure for checkerboard instability. Without the cure for the checkerboard instability $(\mu=0)$, the pressure field exhibits spurious oscillations as shown in Fig. 10. The lack of regularity causes the checkerboard instability and the divergencefree condition is broken locally in compensation for the suppression of spurious oscillations, although the overall behavior of flow velocity is improved. Incidentally, LBM produces nearly identical results to those of ACM in this problem, although it exhibits small spurious oscillations around the two top corners (no figure). The breakdown of divergence-free condition is observed in the LBM computation as well.

\section{CONCLUDING REMARKS}

The present numerical method for INSE using ACE relies on the assumption about the asymptotic behavior of solution for small Mach numbers. The solution after the extinction of acoustic mode is assumed to be in the form of power series of $M a^{2}$, which is the only parameter of the problem. The justification of this anzatz from the dynamical viewpoint remains as an interesting mathematical problem. The numerical method is designed in such a way that the discretization error does not deteriorate the first two terms of the expansion. In order for the straightforward treatment of fourth order accurate approximation 
with respect to space, the simple lattice structure of stencil is employed. Consequently, the boundary value of pressure is computed with sufficient accuracy. The employment of Numerov algorithm is restricted to the grid points next to the boundary in order to avoid the inverse of large matrix. The present study reveals the potential performance of ACM. Although these efforts are rewarded only in the case where the solution has sufficient regularity, the robustness of the scheme is also demonstrated. From practical viewpoint, the second order accurate ACM with second order accurate treatment of curved boundary seems to be desirable. This becomes meaningful only when the acoustic mode is eliminated sufficiently. The present study also adds a contribution to this issue and the treatment of curved boundary is left as one of future subjects. On the other hand, the simple second order accurate ACM, which employs the same compact stencil as that of LBM, yields better results than LBM. Since ACM does not require any kinetic-theory gadgets, its education cost is much less than that of LBM. These facts suggest that drastic improvements of LBM or clear demonstrations of outcomes of kinetic formulation are necessary.

\section{Acknowledgements}

The authors are grateful to Professor Charles-Henri Bruneau of Université Bordeaux I for providing us the numerical data of lid-driven cavity flow. T.O. would like to acknowledge the support from Japan Society for the Promotion of Science (JSPS) under Grant-in-Aid for Scientific Research (C) No.21560173.

\section{A. APPENDIX}

We summarize the finite differential operators for the uniform grid system with the spacing $\epsilon$.

Centered three point formulas

$$
\begin{gathered}
\delta_{x} f_{i, j} \equiv \frac{1}{2 \epsilon}\left[f_{i+1, j}-f_{i-1, j}\right]=\left.\frac{\partial f}{\partial x}\right|_{i, j}+\left.\frac{\epsilon^{2}}{6} \frac{\partial^{3} f}{\partial x^{3}}\right|_{i, j}+O\left(\epsilon^{4}\right), \\
\delta_{y} f_{i, j} \equiv \frac{1}{2 \epsilon}\left[f_{i, j+1}-f_{i, j-1}\right]=\left.\frac{\partial f}{\partial y}\right|_{i, j}+\left.\frac{\epsilon^{2}}{6} \frac{\partial^{3} f}{\partial y^{3}}\right|_{i, j}+O\left(\epsilon^{4}\right), \\
\delta_{x x} f_{i, j} \equiv \frac{1}{\epsilon^{2}}\left[f_{i+1, j}-2 f_{i, j}+f_{i-1, j}\right]=\left.\frac{\partial^{2} f}{\partial x^{2}}\right|_{i, j}+\left.\frac{\epsilon^{2}}{12} \frac{\partial^{4} f}{\partial x^{4}}\right|_{i, j}+O\left(\epsilon^{4}\right), \\
\delta_{y y} f_{i, j} \equiv \frac{1}{\epsilon^{2}}\left[f_{i, j+1}-2 f_{i, j}+f_{i, j-1}\right]=\left.\frac{\partial^{2} f}{\partial y^{2}}\right|_{i, j}+\left.\frac{\epsilon^{2}}{12} \frac{\partial^{4} f}{\partial y^{4}}\right|_{i, j}+O\left(\epsilon^{4}\right), \\
\delta_{x y} f_{i, j} \equiv \delta_{x} \delta_{y} f_{i, j} \equiv \delta_{y} \delta_{x} f_{i, j}=\left.\frac{\partial^{2} f}{\partial x \partial y}\right|_{i, j}+O\left(\epsilon^{2}\right), \\
\delta_{x x y} f_{i, j} \equiv \delta_{x x} \delta_{y} f_{i, j} \equiv \delta_{y} \delta_{x x} f_{i, j}=\left.\frac{\partial^{3} f}{\partial x^{2} \partial y}\right|_{i, j}+O\left(\epsilon^{2}\right),
\end{gathered}
$$




$$
\begin{gathered}
\delta_{x y y} f_{i, j} \equiv \delta_{x} \delta_{y y} f_{i, j} \equiv \delta_{y y} \delta_{x} f_{i, j}=\left.\frac{\partial^{3} f}{\partial x \partial y^{2}}\right|_{i, j}+O\left(\epsilon^{2}\right), \\
\delta_{x x y y} f_{i, j} \equiv \delta_{x x} \delta_{y y} f_{i, j} \equiv \delta_{y y} \delta_{x x} f_{i, j}=\left.\frac{\partial^{4} f}{\partial x^{2} \partial y^{2}}\right|_{i, j}+O\left(\epsilon^{2}\right) .
\end{gathered}
$$

For conciseness, we will express $\delta_{x} u$ as $U_{x}, \delta_{x} v$ as $V_{x}, \delta_{x} P$ as $P_{x}, \delta_{y} u$ as $U_{y}$, $\delta_{x x y} v$ as $V_{x x y}$, and so on. Since no confusion will take place, other definitions are omitted here.

Centered five point formulas

$$
\begin{gathered}
D_{x} f_{i, j}=\frac{1}{12 \epsilon}\left[8\left(f_{i+1, j}-f_{i-1, j}\right)-\left(f_{i+2, j}-f_{i-2, j}\right)\right]=\left.\frac{\partial f}{\partial x}\right|_{i, j}+O\left(\epsilon^{4}\right), \\
D_{x x} f_{i, j}=\frac{1}{12 \epsilon^{2}}\left[16\left(f_{i+1 . j}+f_{i-1, j}\right)-\left(f_{i+2, j}+f_{i-2, j}\right)-30 f_{i, j}\right]=\left.\frac{\partial^{2} f}{\partial x^{2}}\right|_{i, j}+O\left(\epsilon^{4}\right), \\
D_{x x x} f_{i, j}=\frac{1}{2 \epsilon^{3}}\left[f_{i+2, j}-f_{i-2, j}-2\left(f_{i+1, j}-f_{i-1, j}\right)\right]=\left.\frac{\partial^{3} f}{\partial x^{3}}\right|_{i, j}+O\left(\epsilon^{2}\right),
\end{gathered}
$$

One-sided five point formulas

$$
\begin{aligned}
& \dot{D}_{x} f_{i, j}=\frac{1}{12 \epsilon}\left[-3 f_{i-1, j}-10 f_{i, j}+18 f_{i+1, j}-6 f_{i+2, j}+f_{i+3, j}\right]=\left.\frac{\partial f}{\partial x}\right|_{i, j}+\begin{array}{r}
O\left(\epsilon^{4}\right), \\
(128)
\end{array} \\
& \grave{D}_{x} f_{i, j}=-\frac{1}{12 \epsilon}\left[-3 f_{i+1, j}-10 f_{i, j}+18 f_{i-1, j}-6 f_{i-2, j}+f_{i-3, j}\right]=\left.\frac{\partial f}{\partial x}(x, y)\right|_{i, j}+O\left(\epsilon^{4}\right), \\
& \dot{D}_{x x} f_{i, j}=\frac{1}{12 \epsilon}\left[11 f_{i-1, j}-20 f_{i, j}+6 f_{i+1, j}+4 f_{i+2, j}-f_{i+3, j}\right]=\left.\frac{\partial^{2} f}{\partial x^{2}}\right|_{i, j}+O\left(\epsilon^{3}\right), \\
& \grave{D}_{x x} f_{i, j}=\frac{1}{12 \epsilon}\left[11 f_{i+1, j}-20 f_{i, j}+6 f_{i-1, j}+4 f_{i-2, j}-f_{i-3, j}\right]=\left.\frac{\partial^{2} f}{\partial x^{2}}\right|_{i, j}+O\left(\epsilon^{3}\right), \\
& \dot{D}_{x x x} f_{i, j}=\frac{1}{2 \epsilon^{3}}\left[-3 f_{i-1, j}+10 f_{i, j}-12 f_{i+1, j}+6 f_{i+2, j}-f_{i+3, j}\right]=\left.\frac{\partial^{3} f}{\partial x^{3}}\right|_{i, j}+O\left(\epsilon^{2}\right), \\
& \grave{D}_{x x x} f_{i, j}=-\frac{1}{2 \epsilon^{3}}\left[-3 f_{i+1, j}+10 f_{i, j}-12 f_{i-1, j}+6 f_{i-2, j}-f_{i-3, j}\right]=\left.\frac{\partial^{3} f}{\partial x^{3}}\right|_{i, j}+O\left(\epsilon^{2}\right) .
\end{aligned}
$$

The finite-difference operators $D_{y}, D_{y y}, D_{y y y}, \dot{D}_{y}, \dot{D}_{y y y}, \grave{D}_{y}$, and $\grave{D}_{y y y}$ are defined in the same way and their definitions are omitted here. 


\section{References}

[1] P. Asinari and T. Ohwada, "Connection between kinetic methods for fluiddynamic equations and macroscopic finite-difference schemes", Comput. Math. Appl. (in Press).

[2] C.-H. Bruneaux and M. Saad, "The 2D lid-driven cavity problem revisited", Comput. \& Fluids 35, 326-348 (2006).

[3] A. J. Chorin, "A numerical method for solving incompressible viscous flow problems," J. Comput. Phys. 2, 12-26 (1967).

[4] Z. Guo, C. Zheng, and B. Shi, "Lattice Boltzmann equation with multiple effective relaxation times for gaseous microscale flow," Phys. Rev. E 77, 036707 (2008).

[5] D. d'Humières, "Generalized lattice Boltzmann equations", In Rarefied gas dynamics: theory and simulations, B.D. Shizgal and D.P. Weaver (Eds.), Prog. Astronaut. Aeronaut., 159:450, 1992.

[6] X. He and L.-S. Luo, "Lattice Boltzmann model for the incompressible Navier-Stokes Equation," J. Stat. Phys. 88, 927-944 (1997).

[7] X. He, G.D. Doolen, and T. Clark, "Comparison of the lattice Boltzmann method and the artificial compressibility method for Navier-Stokes equations," J. Comput. Phys. 179, 439-451 (2002).

[8] M. Junk and Z. Yang, "Asymptotic analysis of finite difference methods," Appl. Math. Comput. 158, 267-301 (2004).

[9] M. Junk, A. Klar, and L.-S. Luo, "Asymptotic analysis of the lattice Boltzmann equation," J. Computat. Phys. 210, 676-704 (2005).

[10] O. Ladyzhenskaya, The Mathematical Theory of Viscous Incompressible Flow (Fizmatgiz, 1961; English transl., Gordon and Breach, New York, 1969).

[11] P. Lallemand, and L.-S. Luo, "Theory of the lattice Boltzmann method: Dispersion, dissipation, isotropy, Galilean invariance, and stability", Phys. Rev. E, 61:6546, 2000.

[12] I. Moise and M. Ziane, "Renormalization group method. Applications to partial differential equations," J. Dyna. Diff. Eqs. 13, 275-321 (2001).

[13] T. Ohwada and S. Fukata, "Simple derivation of high-resolution schemes for compressible flows by kinetic approach," J. Comput. Phys. 211, 424-447 (2006).

[14] T. Ohwada and P. Asinari, "On the essential role of kinetic theory in numerical methods for fluid-dynamic equations," in Rarefied Gas Dynamics: 26th International Symposium, edited by T. Abe, (American Institute of Physics, 2009), pp.401-408. 
[15] J.D. Ramshaw and V.A. Mousseau, "Accelerated artificial compressibility method for steady-state incompressibility flow calculations," Comput. \& Fluids 18 361-367 (1990).

[16] Y. Qian, D. d'Humières, and P. Lallemand, "Lattice BGK models for Navier-Stokes equation", Europhys. Lett. 17 479-484 (1992).

[17] R. Témam, Navier-Stokes Equations (Revised Edition, North-Holland, Amsterdam, 1979). 


\begin{tabular}{l|lll}
\hline \hline$\epsilon$ & $E[u(\beta=2)]$ & $E[u(\beta=4)]$ & $E[2 u(\beta=2)-u(\beta=4)]$ \\
$\pi / 32$ & $0.21671 \mathrm{E}-02$ & $0.41064 \mathrm{E}-02$ & $0.49013 \mathrm{E}-03$ \\
$\pi / 64$ & $0.56179 \mathrm{E}-03(1.95)$ & $0.11048 \mathrm{E}-02(1.89)$ & $0.40272 \mathrm{E}-04(3.61)$ \\
$\pi / 128$ & $0.14151 \mathrm{E}-03(1.99)$ & $0.28160 \mathrm{E}-03(1.97)$ & $0.29311 \mathrm{E}-05(3.78)$ \\
$\pi / 256$ & $0.35426 \mathrm{E}-04(2.00)$ & $0.70735 \mathrm{E}-04(1.99)$ & $0.22061 \mathrm{E}-06(3.73)$ \\
\hline$\epsilon$ & $E[v(\beta=2)]$ & $E[v(\beta=4)]$ & $E[2 v(\beta=2)-v(\beta=4)]$ \\
$\pi / 32$ & $0.25755 \mathrm{E}-02$ & $0.50410 \mathrm{E}-02$ & $0.49816 \mathrm{E}-03$ \\
$\pi / 64$ & $0.65540 \mathrm{E}-03(1.97)$ & $0.13004 \mathrm{E}-02(1.95)$ & $0.39386 \mathrm{E}-04(3.66)$ \\
$\pi / 128$ & $0.16470 \mathrm{E}-03(1.99)$ & $0.32868 \mathrm{E}-03(1.98)$ & $0.27622 \mathrm{E}-05(3.83)$ \\
$\pi / 256$ & $0.41228 \mathrm{E}-04(2.00)$ & $0.82406 \mathrm{E}-04(2.00)$ & $0.19643 \mathrm{E}-06(3.81)$ \\
\hline$\epsilon$ & $E[P(\beta=2)]$ & $E[P(\beta=4)]$ & $E[2 P(\beta=2)-P(\beta=4)]$ \\
$\pi / 32$ & $0.11282 \mathrm{E}-02$ & $0.24926 \mathrm{E}-02$ & $0.35484 \mathrm{E}-03$ \\
$\pi / 64$ & $0.25806 \mathrm{E}-03(2.13)$ & $0.52368 \mathrm{E}-03(2.25)$ & $0.27835 \mathrm{E}-04(3.67)$ \\
$\pi / 128$ & $0.63296 \mathrm{E}-04(2.03)$ & $0.12686 \mathrm{E}-03(2.05)$ & $0.19420 \mathrm{E}-05(3.84)$ \\
$\pi / 256$ & $0.15747 \mathrm{E}-04(2.01)$ & $0.31498 \mathrm{E}-04(2.01)$ & $0.13847 \mathrm{E}-06(3.81)$ \\
\hline \hline
\end{tabular}

Table 1: The $L^{1}$ error vs. $\epsilon$ at $t=60$ in the problem of generalized Taylor-Green problem for $\nu=0.001$. The value in each parenthesis indicates the local convergence rate. 


\begin{tabular}{l|lll}
\hline \hline$\epsilon$ & $E[u(\beta=2)]$ & $E[u(\beta=4)]$ & $E[2 u(\beta=2)-u(\beta=4)]$ \\
$\pi / 32$ & $0.18944 \mathrm{E}-02$ & $0.35741 \mathrm{E}-02$ & $0.48449 \mathrm{E}-03$ \\
$\pi / 64$ & $0.56046 \mathrm{E}-03(1.76)$ & $0.10941 \mathrm{E}-02(1.71)$ & $0.62921 \mathrm{E}-04(2.94)$ \\
$\pi / 128$ & $0.14289 \mathrm{E}-03(1.97)$ & $0.28298 \mathrm{E}-03(1.95)$ & $0.10834 \mathrm{E}-04(2.54)$ \\
$\pi / 256$ & $0.35735 \mathrm{E}-04(2.00)$ & $0.71036 \mathrm{E}-04(1.99)$ & $0.24187 \mathrm{E}-05(2.16)$ \\
\hline$\epsilon$ & $E[v(\beta=2)]$ & $E[v(\beta=4)]$ & $E[2 v(\beta=2)-v(\beta=4)]$ \\
$\pi / 32$ & $0.24440 \mathrm{E}-02$ & $0.47557 \mathrm{E}-02$ & $0.47049 \mathrm{E}-03$ \\
$\pi / 64$ & $0.65619 \mathrm{E}-03(1.90)$ & $0.12991 \mathrm{E}-02(1.87)$ & $0.67371 \mathrm{E}-04(2.80)$ \\
$\pi / 128$ & $0.16544 \mathrm{E}-03(1.99)$ & $0.32950 \mathrm{E}-03(1.98)$ & $0.11018 \mathrm{E}-04(2.61)$ \\
$\pi / 256$ & $0.41383 \mathrm{E}-04(2.00)$ & $0.82555 \mathrm{E}-04(2.00)$ & $0.24121 \mathrm{E}-05(2.19)$ \\
\hline$\epsilon$ & $E[P(\beta=2)]$ & $E[P(\beta=4)]$ & $E[2 P(\beta=2)-P(\beta=4)]$ \\
$\pi / 32$ & $0.19197 \mathrm{E}-02$ & $0.33617 \mathrm{E}-02$ & $0.61604 \mathrm{E}-03$ \\
$\pi / 64$ & $0.43247 \mathrm{E}-03(2.15)$ & $0.68596 \mathrm{E}-03(2.29)$ & $0.20753 \mathrm{E}-03(1.57)$ \\
$\pi / 128$ & $0.10539 \mathrm{E}-03(2.04)$ & $0.16204 \mathrm{E}-03(2.08)$ & $0.54912 \mathrm{E}-04(1.92)$ \\
$\pi / 256$ & $0.26156 \mathrm{E}-04(2.01)$ & $0.39941 \mathrm{E}-04(2.02)$ & $0.13885 \mathrm{E}-04(1.98)$ \\
\hline \hline
\end{tabular}

Table 2: The $L^{1}$ error vs. $\epsilon$ at $t=60$ in the problem of generalized Taylor-Green vortices for $\nu=0.001$ (2nd order accurate scheme).

\begin{tabular}{l|lll}
\hline \hline$\epsilon$ & $E[u(\beta=2)]$ & $E[u(\beta=4)]$ & $E[2 u(\beta=2)-u(\beta=4)]$ \\
$\pi / 32$ & $0.16219 \mathrm{E}-02$ & $0.32445 \mathrm{E}-02$ & $0.11181 \mathrm{E}-03$ \\
$\pi / 64$ & $0.39836 \mathrm{E}-03(2.03)$ & $0.79465 \mathrm{E}-03(2.03)$ & $0.77579 \mathrm{E}-05(3.85)$ \\
$\pi / 128$ & $0.98902 \mathrm{E}-04(2.01)$ & $0.19741 \mathrm{E}-03(2.01)$ & $0.74768 \mathrm{E}-06(3.38)$ \\
$\pi / 256$ & $0.24654 \mathrm{E}-04(2.00)$ & $0.49250 \mathrm{E}-04(2.00)$ & $0.81806 \mathrm{E}-07(3.19)$ \\
\hline$\epsilon$ & $E[v(\beta=2)]$ & $E[v(\beta=4)]$ & $E[2 v(\beta=2)-v(\beta=4)]$ \\
$\pi / 32$ & $0.20829 \mathrm{E}-02$ & $0.42087 \mathrm{E}-02$ & $0.14459 \mathrm{E}-03$ \\
$\pi / 64$ & $0.51354 \mathrm{E}-03(2.02)$ & $0.10289 \mathrm{E}-02(2.03)$ & $0.73480 \mathrm{E}-05(4.30)$ \\
$\pi / 128$ & $0.12788 \mathrm{E}-03(2.01)$ & $0.25579 \mathrm{E}-03(2.01)$ & $0.58774 \mathrm{E}-06(3.64)$ \\
$\pi / 256$ & $0.31933 \mathrm{E}-04(2.00)$ & $0.63858 \mathrm{E}-04(2.00)$ & $0.57893 \mathrm{E}-07(3.34)$ \\
\hline$\epsilon$ & $E[P(\beta=2)]$ & $E[P(\beta=4)]$ & $E[2 P(\beta=2)-P(\beta=4)]$ \\
$\pi / 32$ & $0.11783 \mathrm{E}-02$ & $0.23611 \mathrm{E}-02$ & $0.85262 \mathrm{E}-04$ \\
$\pi / 64$ & $0.28480 \mathrm{E}-03(2.05)$ & $0.56931 \mathrm{E}-03(2.05)$ & $0.53759 \mathrm{E}-05(3.99)$ \\
$\pi / 128$ & $0.70452 \mathrm{E}-04(2.02)$ & $0.14080 \mathrm{E}-03(2.02)$ & $0.47729 \mathrm{E}-06(3.49)$ \\
$\pi / 256$ & $0.17555 \mathrm{E}-04(2.00)$ & $0.35092 \mathrm{E}-04(2.00)$ & $0.53583 \mathrm{E}-07(3.16)$ \\
\hline \hline
\end{tabular}

Table 3: The $L^{1}$ error vs. $\epsilon$ at $t=60$ in the problem of generalized Taylor-Green problem for $\nu=0.1$. 


\begin{tabular}{l|llll}
\hline \hline$\epsilon$ & $E[u(\beta=2)]$ & $E[u(\beta=4)]$ & $E[2 u(\beta=2)-u(\beta=4)]$ & $E[u]$ forLBM \\
$\pi / 32$ & $0.16506 \mathrm{E}-02$ & $0.32740 \mathrm{E}-02$ & $0.25524 \mathrm{E}-03$ & $0.43693 \mathrm{E}-02$ \\
$\pi / 64$ & $0.40394 \mathrm{E}-03(2.03)$ & $0.79929 \mathrm{E}-03(2.03)$ & $0.60917 \mathrm{E}-04(2.07)$ & $0.10786 \mathrm{E}-02(2.02)$ \\
$\pi / 128$ & $0.10022 \mathrm{E}-03(2.01)$ & $0.19844 \mathrm{E}-03(2.01)$ & $0.14762 \mathrm{E}-04(2.04)$ & $0.26914 \mathrm{E}-03(2.00)$ \\
$\pi / 256$ & $0.24979 \mathrm{E}-04(2.00)$ & $0.49501 \mathrm{E}-04(2.00)$ & $0.36220 \mathrm{E}-05(2.03)$ & $0.67259 \mathrm{E}-04(2.00)$ \\
\hline$\epsilon$ & $E[v(\beta=2)]$ & $E[v(\beta=4)]$ & $E[2 v(\beta=2)-v(\beta=4)]$ & $E[v]$ for LBM \\
$\pi / 32$ & $0.21159 \mathrm{E}-02$ & $0.42473 \mathrm{E}-02$ & $0.25380 \mathrm{E}-03$ & $0.53746 \mathrm{E}-02$ \\
$\pi / 64$ & $0.52014 \mathrm{E}-03(2.02)$ & $0.10345 \mathrm{E}-02(2.04)$ & $0.59323 \mathrm{E}-04(2.10)$ & $0.13362 \mathrm{E}-02(2.01)$ \\
$\pi / 128$ & $0.12944 \mathrm{E}-03(2.01)$ & $0.25703 \mathrm{E}-03(2.01)$ & $0.14552 \mathrm{E}-04(2.03)$ & $0.33387 \mathrm{E}-03(2.00)$ \\
$\pi / 256$ & $0.32316 \mathrm{E}-04(2.00)$ & $0.64154 \mathrm{E}-04(2.00)$ & $0.35953 \mathrm{E}-05(2.02)$ & $0.83463 \mathrm{E}-04(2.00)$ \\
\hline$\epsilon$ & $E[P(\beta=2)]$ & $E[P(\beta=4)]$ & $E[2 P(\beta=2)-P(\beta=4)]$ & $E[P]$ for LBM \\
$\pi / 32$ & $0.20663 \mathrm{E}-02$ & $0.32585 \mathrm{E}-02$ & $0.92971 \mathrm{E}-03$ & $0.93929 \mathrm{E}-02$ \\
$\pi / 64$ & $0.50254 \mathrm{E}-03(2.04)$ & $0.78416 \mathrm{E}-03(2.05)$ & $0.23234 \mathrm{E}-03(2.00)$ & $0.22371 \mathrm{E}-02(2.07)$ \\
$\pi / 128$ & $0.12465 \mathrm{E}-03(2.01)$ & $0.19394 \mathrm{E}-03(2.02)$ & $0.58047 \mathrm{E}-04(2.00)$ & $0.55336 \mathrm{E}-03(2.02)$ \\
$\pi / 256$ & $0.31088 \mathrm{E}-04(2.00)$ & $0.48343 \mathrm{E}-04(2.00)$ & $0.14495 \mathrm{E}-04(2.00)$ & $0.13800 \mathrm{E}-03(2.00)$ \\
\hline \hline
\end{tabular}

Table 4: The $L^{1}$ error vs. $\epsilon$ at $t=60$ in the problem of generalized Taylor-Green vortices for $\nu=0.1$ (2nd order accurate scheme and LBM).

\begin{tabular}{l|lll}
\hline \hline$\epsilon$ & $E[u(\beta=2)]$ & $E[u(\beta=4)]$ & $E[2 u(\beta=2)-u(\beta=4)]$ \\
$\pi / 32$ & $0.19312 \mathrm{E}-01$ & $0.37429 \mathrm{E}-01$ & $0.79410 \mathrm{E}-02$ \\
$\pi / 64$ & $0.48916 \mathrm{E}-02(1.98)$ & $0.97529 \mathrm{E}-02(1.94)$ & $0.48152 \mathrm{E}-03(4.04)$ \\
$\pi / 128$ & $0.12253 \mathrm{E}-02(2.00)$ & $0.24493 \mathrm{E}-02(1.99)$ & $0.29419 \mathrm{E}-04(4.03)$ \\
$\pi / 256$ & $0.30648 \mathrm{E}-03(2.00)$ & $0.61288 \mathrm{E}-03(2.00)$ & $0.18272 \mathrm{E}-05(4.01)$ \\
$\pi / 512$ & $0.76630 \mathrm{E}-04(2.00)$ & $0.15325 \mathrm{E}-03(2.00)$ & $0.11420 \mathrm{E}-06$ \\
\hline$\epsilon$ & $E[v(\beta=2)]$ & $E[v(\beta=4)]$ & $E[2 v(\beta=2)-v(\beta=4)]$ \\
$\pi / 32$ & $0.24663 \mathrm{E}-01$ & $0.47630 \mathrm{E}-01$ & $0.12847 \mathrm{E}-01$ \\
$\pi / 64$ & $0.61884 \mathrm{E}-02(1.99)$ & $0.12388 \mathrm{E}-01(1.94)$ & $0.83582 \mathrm{E}-03(3.94)$ \\
$\pi / 128$ & $0.15445 \mathrm{E}-02(2.00)$ & $0.30910 \mathrm{E}-02(2.00)$ & $0.51849 \mathrm{E}-04(4.01)$ \\
$\pi / 256$ & $0.38593 \mathrm{E}-03(2.00)$ & $0.77201 \mathrm{E}-03(2.00)$ & $0.32301 \mathrm{E}-05(4.00)$ \\
$\pi / 512$ & $0.96470 \mathrm{E}-04(2.00)$ & $0.19295 \mathrm{E}-03(2.00)$ & $0.20188 \mathrm{E}-06$ \\
\hline$\epsilon$ & $E[P(\beta=2)]$ & $E[P(\beta=4)]$ & $E[2 P(\beta=2)-P(\beta=4)]$ \\
$\pi / 32$ & $0.62879 \mathrm{E}-02$ & $0.11829 \mathrm{E}-01$ & $0.24083 \mathrm{E}-02$ \\
$\pi / 64$ & $0.16145 \mathrm{E}-02(1.96)$ & $0.32084 \mathrm{E}-02(1.88)$ & $0.14579 \mathrm{E}-03(4.05)$ \\
$\pi / 128$ & $0.40530 \mathrm{E}-03(1.99)$ & $0.80962 \mathrm{E}-03(1.99)$ & $0.89772 \mathrm{E}-05(4.02)$ \\
$\pi / 256$ & $0.10143 \mathrm{E}-03(2.00)$ & $0.20278 \mathrm{E}-03(2.00)$ & $0.58211 \mathrm{E}-06(3.95)$ \\
$\pi / 512$ & $0.25358 \mathrm{E}-04(2.00)$ & $0.50712 \mathrm{E}-04(2.00)$ & $0.36382 \mathrm{E}-07$ \\
\hline \hline
\end{tabular}

Table 5: The $L^{1}$ error vs. $\epsilon$ at $t=30$ in the problem of formation of two rolls $(\nu=0.01)$. The value in each parenthesis indicates the local convergence rate. 


\begin{tabular}{l|lll}
\hline \hline$\epsilon$ & $E[u(\beta=2)]$ & $E[u(\beta=4)]$ & $E[2 u(\beta=2)-u(\beta=4)]$ \\
$\pi / 32$ & $0.19207 \mathrm{E}-01$ & $0.33282 \mathrm{E}-01$ & $0.19447 \mathrm{E}-01$ \\
$\pi / 64$ & $0.50724 \mathrm{E}-02(1.92)$ & $0.10016 \mathrm{E}-01(1.73)$ & $0.18696 \mathrm{E}-02(3.38)$ \\
$\pi / 128$ & $0.12501 \mathrm{E}-02(2.02)$ & $0.25043 \mathrm{E}-02(2.00)$ & $0.12269 \mathrm{E}-03(3.93)$ \\
$\pi / 256$ & $0.31162 \mathrm{E}-03(2.00)$ & $0.62366 \mathrm{E}-03(2.01)$ & $0.77538 \mathrm{E}-05(3.98)$ \\
$\pi / 512$ & $0.77864 \mathrm{E}-04(2.00)$ & $0.15575 \mathrm{E}-03(2.00)$ & $0.48461 \mathrm{E}-06$ \\
\hline$\epsilon$ & $E[v(\beta=2)]$ & $E[v(\beta=4)]$ & $E[2 v(\beta=2)-v(\beta=4)]$ \\
$\pi / 32$ & $0.25326 \mathrm{E}-01$ & $0.37347 \mathrm{E}-01$ & $0.26227 \mathrm{E}-01$ \\
$\pi / 64$ & $0.73955 \mathrm{E}-02(1.78)$ & $0.14375 \mathrm{E}-01(1.38)$ & $0.30242 \mathrm{E}-02(3.12)$ \\
$\pi / 128$ & $0.18744 \mathrm{E}-02(1.98)$ & $0.37429 \mathrm{E}-02(1.94)$ & $0.20693 \mathrm{E}-03(3.87)$ \\
$\pi / 256$ & $0.46920 \mathrm{E}-03(2.00)$ & $0.93839 \mathrm{E}-03(2.00)$ & $0.13259 \mathrm{E}-04(3.96)$ \\
$\pi / 512$ & $0.11734 \mathrm{E}-03(2.00)$ & $0.23467 \mathrm{E}-03(2.00)$ & $0.82871 \mathrm{E}-06$ \\
\hline$\epsilon$ & $E[P(\beta=2)]$ & $E[P(\beta=4)]$ & $E[2 P(\beta=2)-P(\beta=4)]$ \\
$\pi / 32$ & $0.39390 \mathrm{E}-02$ & $0.62971 \mathrm{E}-02$ & $0.34197 \mathrm{E}-02$ \\
$\pi / 64$ & $0.11306 \mathrm{E}-02(1.80)$ & $0.21944 \mathrm{E}-02(1.52)$ & $0.33323 \mathrm{E}-03(3.36)$ \\
$\pi / 128$ & $0.27904 \mathrm{E}-03(2.02)$ & $0.55702 \mathrm{E}-03(1.98)$ & $0.22034 \mathrm{E}-04(3.92)$ \\
$\pi / 256$ & $0.69489 \mathrm{E}-04(2.01)$ & $0.13901 \mathrm{E}-03(2.00)$ & $0.14314 \mathrm{E}-05(3.94)$ \\
$\pi / 512$ & $0.17362 \mathrm{E}-04(2.00)$ & $0.34726 \mathrm{E}-04(2.00)$ & $0.89462 \mathrm{E}-07$ \\
\hline \hline
\end{tabular}

Table 6: The $L^{1}$ error vs. $\epsilon$ at $t=30$ in the problem of formation of two rolls $(\nu=0.001)$. The value in each parenthesis indicates the local convergence rate. 


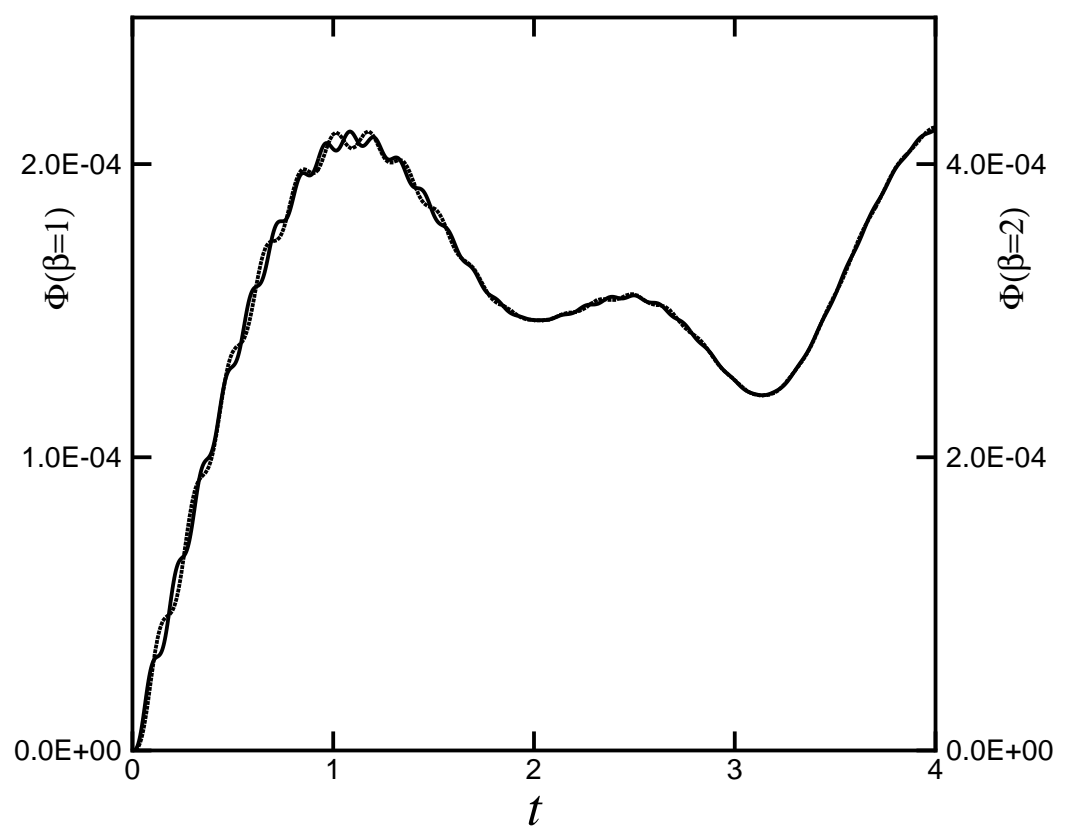

Figure 1: The time history of $L^{1}$ error from $t=0$ to $t=1$ in the problem of generalized Taylor-Green vortices $(\nu=0.001)$. 




Figure 2: The time history of $L^{1}$ error from $t=4$ to $t=15$ in the problem of generalized Taylor-Green vortices $(\nu=0.001)$. 


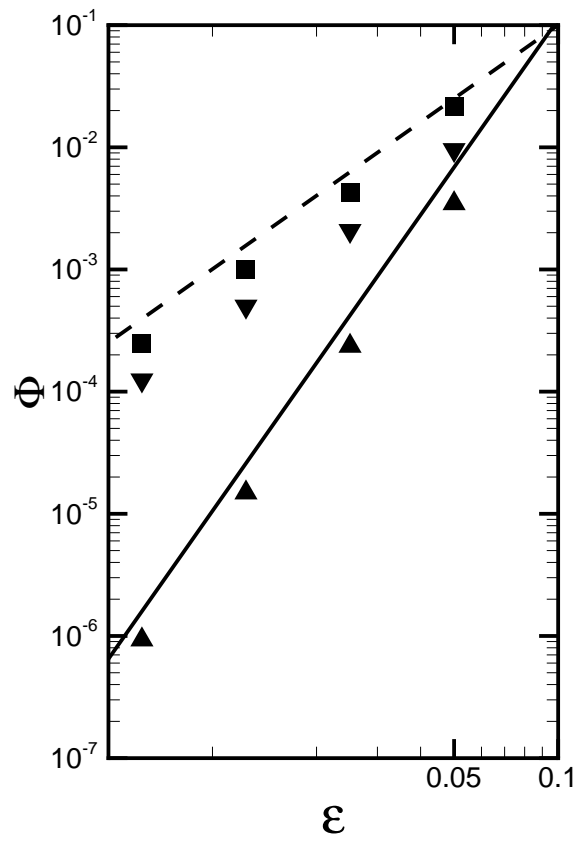

Figure 3: The flow field at $t=30$ in the problem of formation of two rolls $(\nu=0.01)$; upper: streamlines; lower: pressure. 


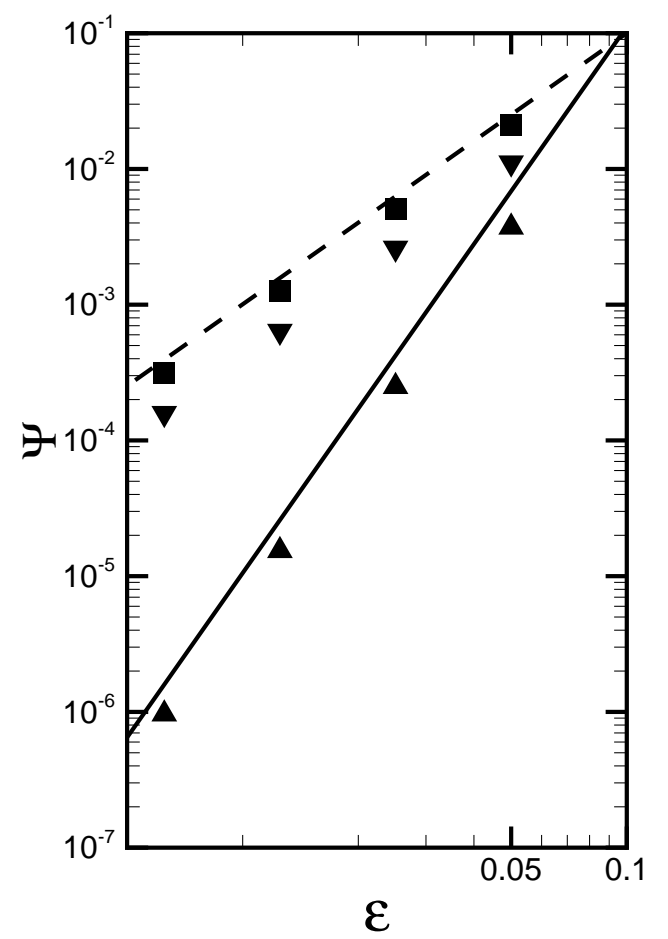

Figure 4: The flow field at $t=30$ in the problem of formation of two rolls $(\nu=0.001)$; upper: streamlines; lower: pressure. 


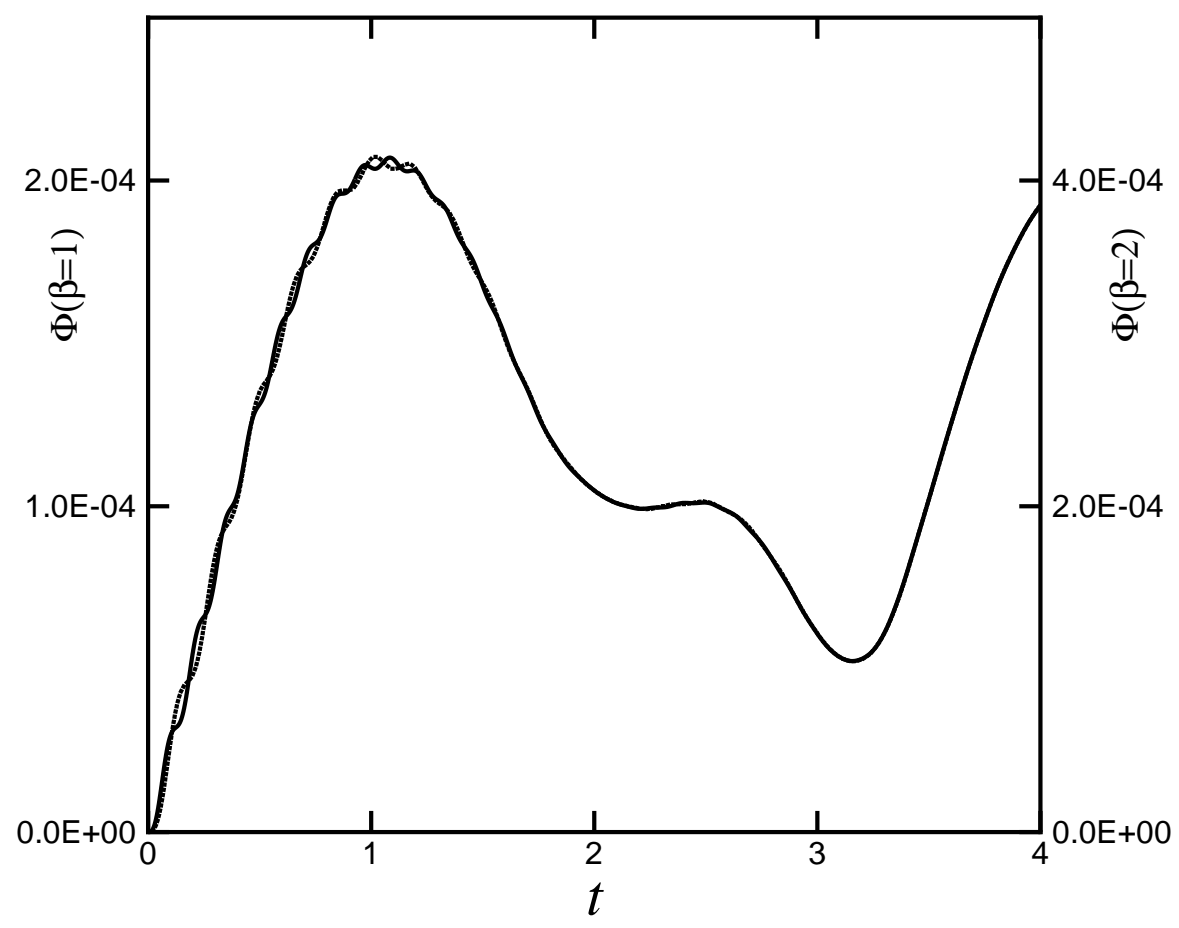

Figure 5: Comparison of flow velocity at $t=30$ in the problem of formation of two rolls ( $\nu=0.01$ and $\epsilon=\pi / 64$ ); upper: $u$ contours; lower: $v$ contours. The dashed lines, the dashdot lines, and the dotted lines indicate the result for $\beta=2$, that for $\beta=4$, and the refined solution generated from these two results. The solid lines indicate the reference solution (the refined solution for $\epsilon=\pi / 512)$. 

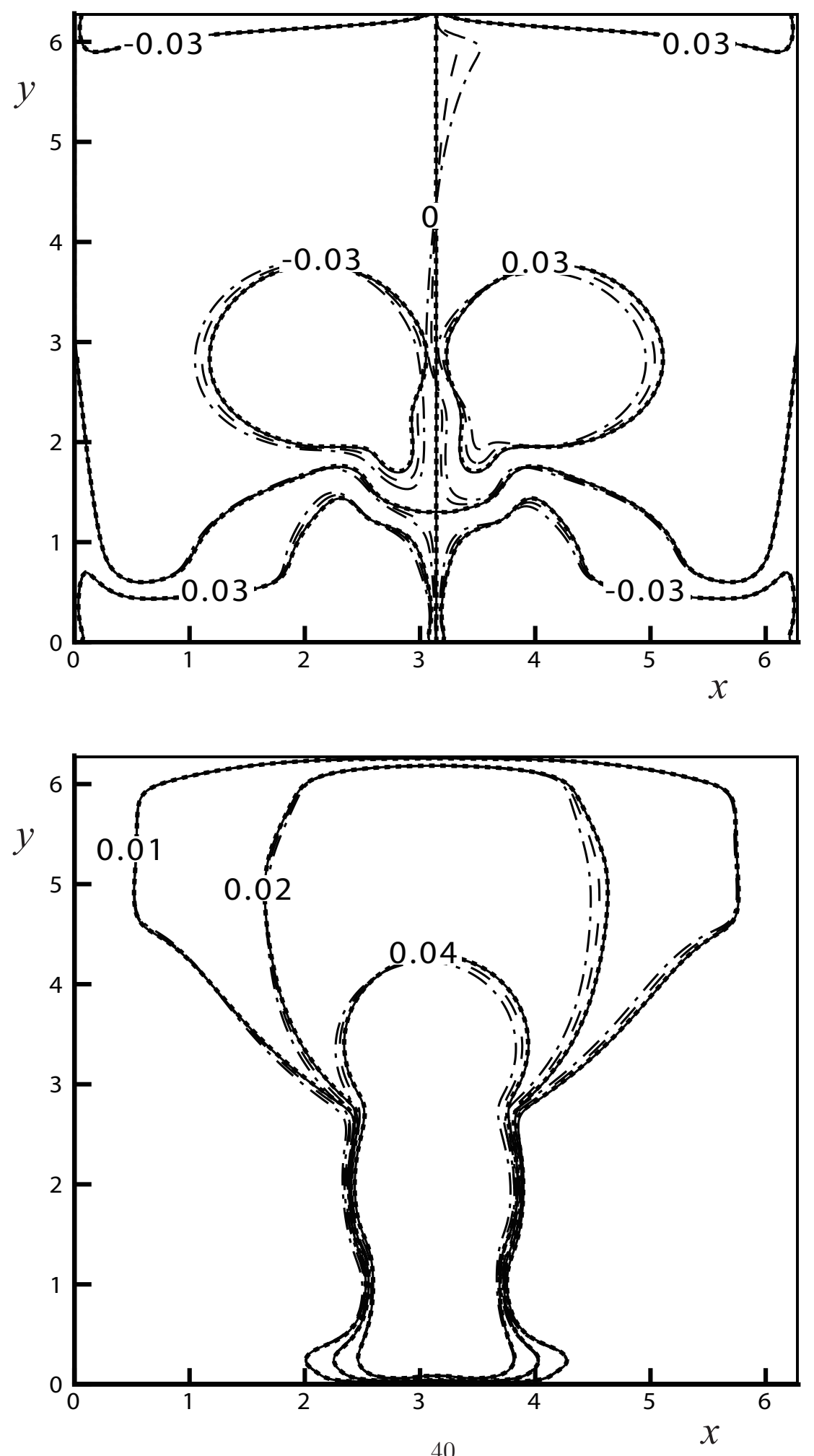

Figure 6: Comparison of flow velocity at $t=30$ in the problem of formation of two rolls ( $\nu=0.001$ and $\epsilon=\pi / 128)$; upper: $u$ contours; lower: $v$ contours. The dashed lines, the dashdot lines, and the dotted lines indicate the result for $\beta=2$, that for $\beta=4$, and the refined solution generated from these two results. The solid lines indicate the reference solution (the refined solution for $\epsilon=\pi / 512)$. 


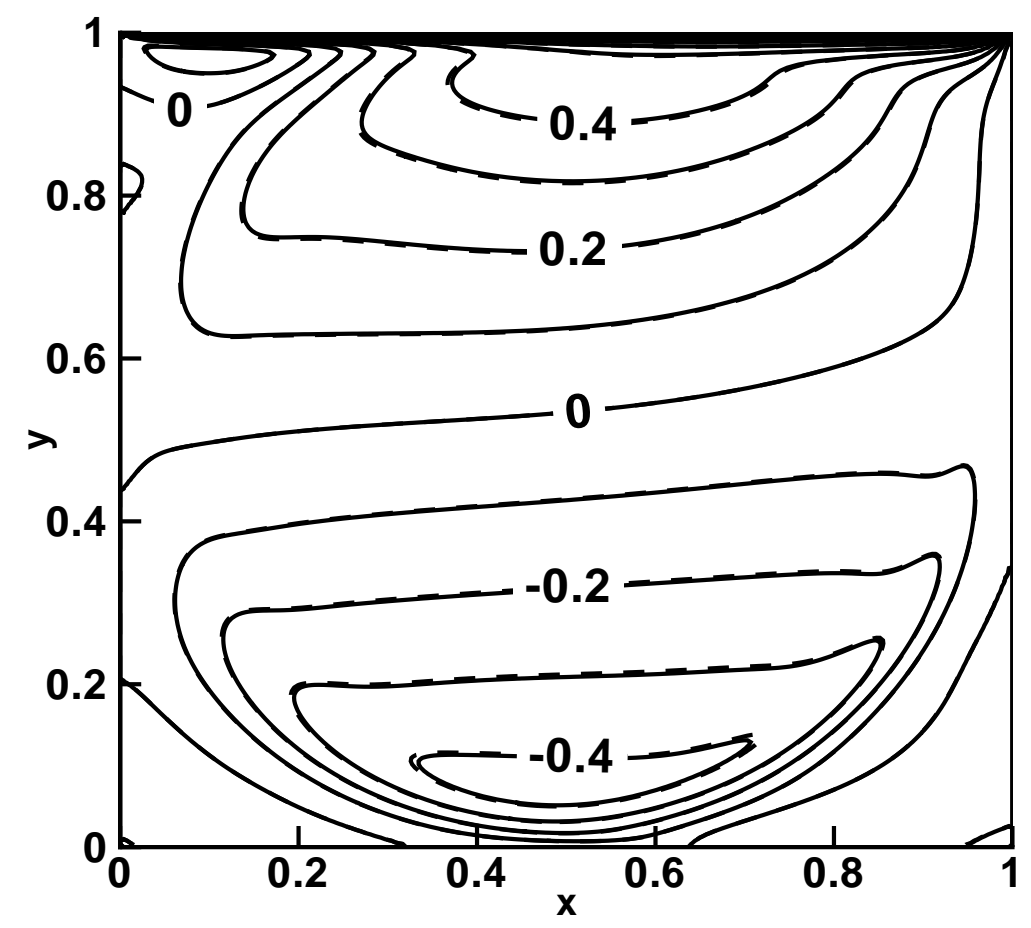

Figure 7: Comparison of flow fields in the problem of lid-driven cavity flow $(\operatorname{Re}=5000): u$ field. The solid lines indicate the ACM solution $\left(\beta=2,\left(N_{x}, N_{y}\right)=(256,256)\right.$ and the dashed lines indicate the reference solution of Ref. [2]. 


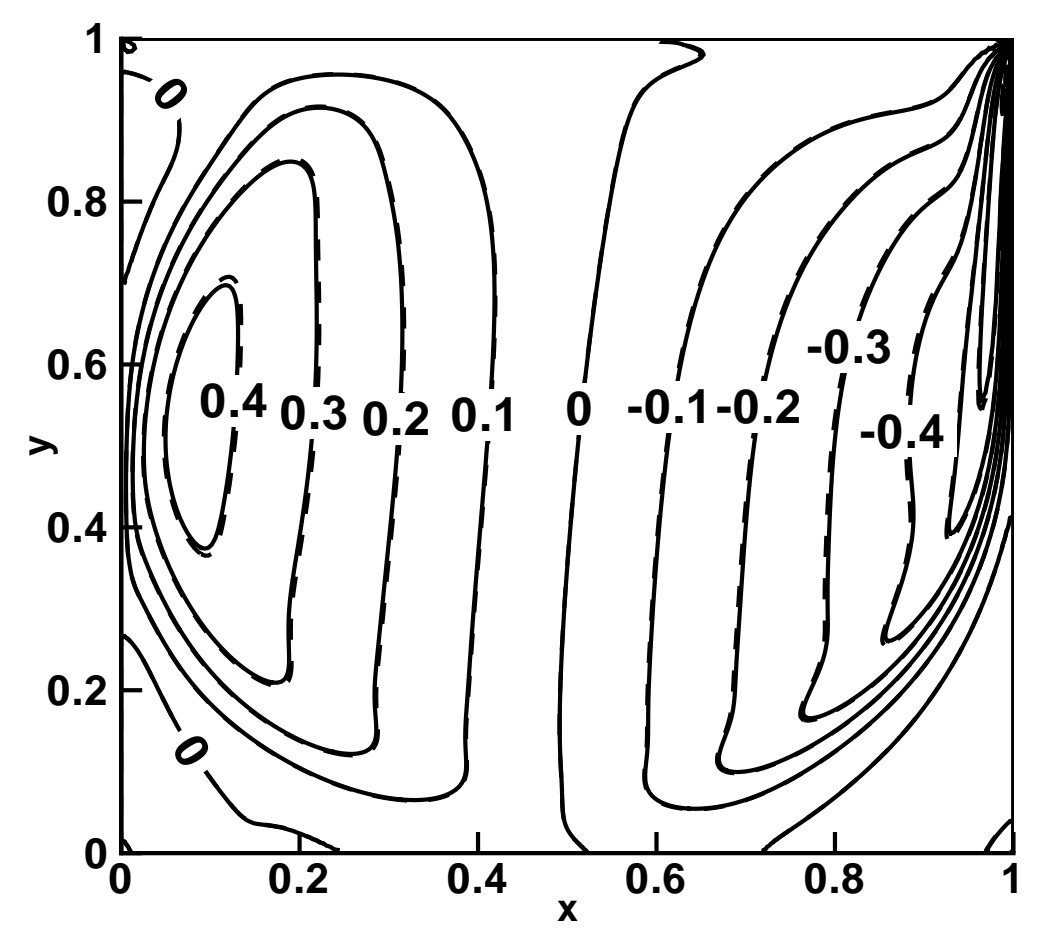

Figure 8: Comparison of flow fields in the problem of lid-driven cavity flow $(\operatorname{Re}=5000): v$ field. 


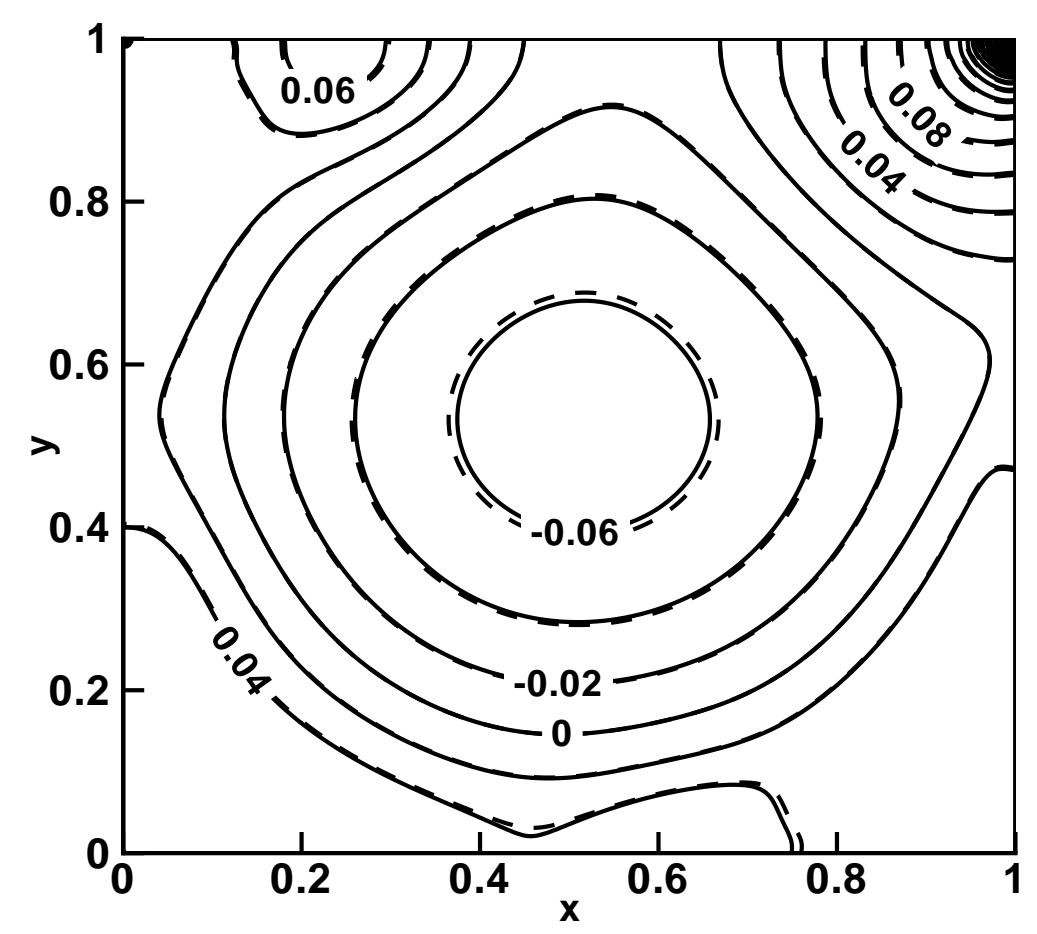

Figure 9: Comparison of flow fields in the problem of lid-driven cavity flow $(\operatorname{Re}=5000): P$ field. 


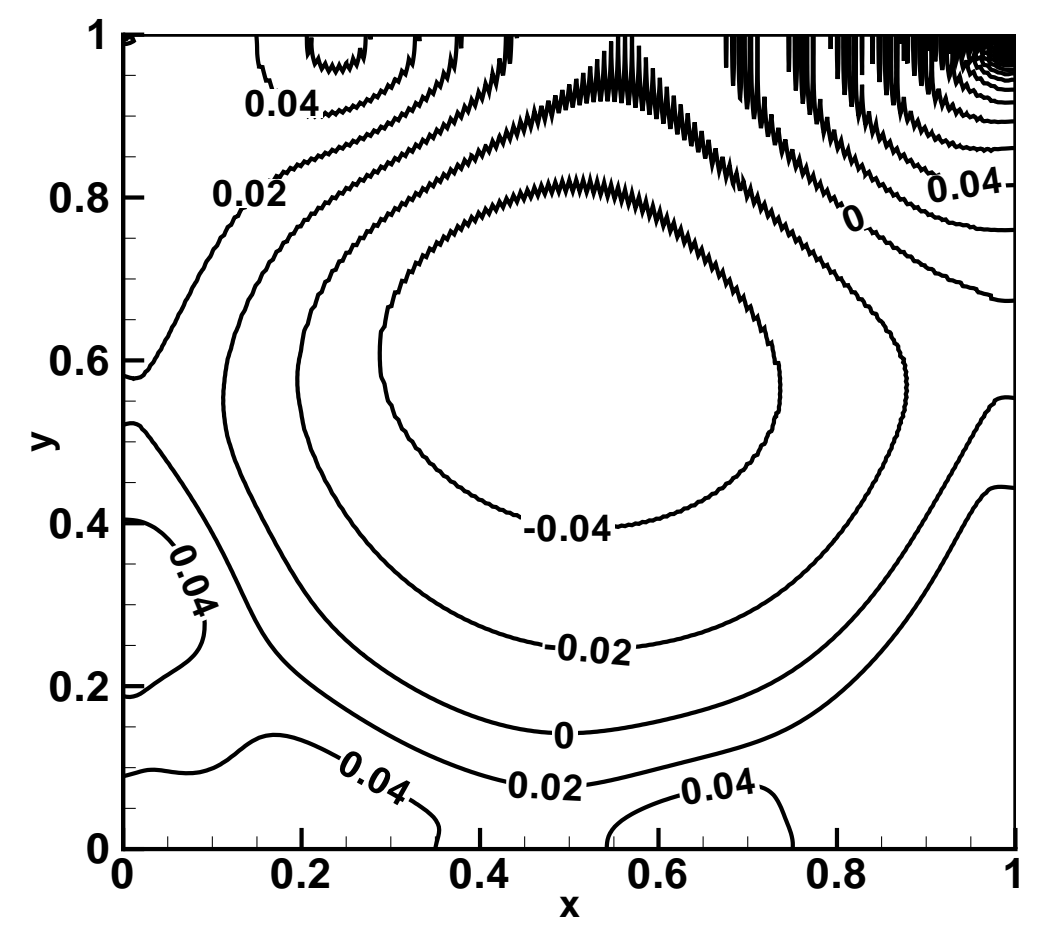

Figure 10: Pressure field for $\mu=0(\operatorname{Re}=5000, \epsilon=1 / 256)$. 